\title{
A device for assessment of the contractile force of different groups of muscles of upper and lower limbs
}

\author{
Dr. Ahmed Abdelkader Guneid ${ }^{(1)}$ \\ Dr. Shimaa Mohammed Ashour Elkhwaga ${ }^{(2)}$ \\ Dr. Alaa M. Khalil ${ }^{(3)}$ \\ Dr. Elsayed Aly Mohamed Metwally ${ }^{(4)}$
}

\begin{abstract}
:
Background: There are no currently reliable devices for the appropriate calculation of the force exhibited by the muscles in different parts of the body. A device is unique in measuring the contractile force of different groups of muscles of the upper and lower limb is invented.
\end{abstract}

Aim of the work: The aim of this study was to examine of a new device for the assessment of the contractile force of different groups of muscles of upper and lower limbs.

Materials and methods: 270 subjects of both sexes at different age group and different body mass index; 135 athletics and 135 non-athletics were assessed on two sessions 2 weeks apart. To aid implementation we have created a new device with freely available software to extract data of the device in $\mathrm{Kg}$ and convert it into newton. The contractile force of different groups of muscles of the upper limb was measured at 2 sessions by 3 professional assessors. Statistical analysis using IBM SPSS software package version 20.0. Quantitative data were described using mean and standard deviation for normally distributed data. Comparison between two independent population were done using independent t-test while more than two population were analyzed F-test (ANOVA) to be used.

Results: Comparison of the contractile force of different groups of muscles of the upper and lower limbs between the 1st and 2nd measurements revealed that there was a highly significant positive correlation, the kappa in all variables was more than 0.70, indicating a high association of the two measurements. The new device has an excellent for most measures of force in athletics and nonathletics at different age groups.

Conclusion: the new device is assessment of the contractile force of different groups of muscles of the upper and lower limbs.

\section{Introduction:}

Skeletal muscles rarely work by themselves to achieve movements in the body. More often they work in groups to produce precise movements[9]. The muscle that produces any particular movement of the body is known as an agonist or prime mover. The agonist always pairs with an antagonist muscle that produces the opposite effect on the same bones. In addition to the agonist/antagonist pairing, other muscles work to support the movements of the agonist. Synergists are muscles that help to stabilize a movement and reduce extraneous movements. They are usually found in regions near the agonist and often connect to the same bones. Because skeletal muscles move the insertion closer to the immobile origin, fixator muscles assist in movement by holding the origin stable [15].

Two important components of muscle function are; muscle strength which is the peak force that a muscle group can produce and muscle power which is how rapidly that force can be produced [15]. Muscular weakness, as a component of muscle function, is an impairment that is commonly observed in clinical populations and has been widely documented to impact upon physical function[16]. Muscle power has previously been quantified by calculating the rate of force development (RFD), which is calculated by measuring the change in force over a certain period of time ( $\Delta$ force/ $\Delta$ time), usually during an isometric contraction [5]. Previous literature assumed that reduced muscle power, commonly associated with old age, may contribute to reduced physical

(1) Professor Anatomy, at Department Anatomy, Faculty of Medicine, Alexandria University

(2) Lecturer at Department Of Health Sciences, Faculty of Physical Education for Girls, Alexandria University.

(3) Lecturer at Department Basic Sciences, Faculty of Engineering, Pharos University in Alexandria.

(4) Assistant Professor at Department Anatomy, Faculty of Medicine, Alexandria University. 
function and an increased risk of falls in a range of clinical populations. Assessments of muscle power may be useful in clinical settings for identifying individuals at risk of falls and functional limitations [1].

There are varying methods utilized to calculate RFD from isometric contractions, commonly used methods involve calculating the change in force over the change in time with discrete time intervals from the onset of contraction to 30,50 or $100 \mathrm{~ms}$ [13]. Other methods of calculating RFD involve examining successive time intervals (e.g. 5ms) during the initial rise in force to determine the peak RFD across the trial or examining the RFD between percentages of the peak force (e.g. between 30 and $60 \%$ of peak force) [4].

laboratory-based dynamometry is used in the assessment of muscle strength and power, but limitation of laboratory-based dynamometers is that they are expensive and difficult preventing their use as a clinically-suitable device for routine patient assessment [8]. Linear position transducers can be used to assess dynamic muscle power however the cost, availability, timeconsuming nature, and difficulty of implementation of such assessments may limit their use in clinical settings [3]. This study aimed to determine the concurrent of a new device to assess the contractile force of different groups of muscles of the upper and lower limbs.

\section{Materials and Methods:}

\section{Research Methodology:}

The researchers used a descriptive approach due to its suitability to the nature of the research.

\section{Participants:}

Duration of the study:

The study lasted about seven months in the period from 28/10/2018 to 28/5/2019 distributed as follows:

- The pilot study was conducted from $28 / 10 / 2018$ to $1 / 11 / 2018$.

- The first basic study was conducted in the period from 2/11/2018 to 2/3/2019.

- The second basic study was conducted in the period from 3/3/2019 to29/5/2019.

\section{Measurements used:}

\section{A. Anthropometric Measurements:}

- Measuring the total length of the body to the nearest $(\mathrm{cm})$

- Measuring weight to nearest $(\mathrm{kg})$

- Find the Body Mass Index (BMI) Body Mass Index, obtained from the following equation: weight $(\mathrm{kg})$ / square length (meters).

\section{B - Measurements of muscle strength of the upper limb muscles:}

- pronation Forearm right / left (Newton).

- supination Forearm right / left (Newton).

- flexion wrist right / left (Newton).

- extension wrist right / left (Newton).

- flexion Elbow Right / left (Newton).

- shoulder Right / left (Newton).

\section{C - Measurements of muscle strength of the lower limb muscles:}

- planterflexion Foot Right / left (Newton).

- dorsiflexion Foot Right / left (Newton).

- eversion Foot Right / left (Newton).

- inversion Foot Right / left (Newton).

\section{The pilot Study:}

Pilot study was done in the club of the Sports Foundation and Ezz El_Dekheila in Alexandria - Dar Mohammed Ragab for the care of the elderly in Alexandria - the school of Omar Lotfy and Mohammed Korium school in Alexandria from 28/10/2018 to 1/11/2018 with the aim of taking approval from the administrative personnel for the participants in the study sample. 
The basic study:

The contractile force of different groups of muscles of 270 subjects of both sexes at different age group and different body mass index; 135 athletics and 135 non-athletics were assessed on two sessions 2 weeks apart. The demographic distribution and BMI of the participants is shown in (table. 1 and 2).

Table (1): Age distribution among the studied groups.

\begin{tabular}{l|c|c|c|c|c}
\hline \multicolumn{1}{c|}{ Age } & $\mathrm{N}$ & Minimum & Maximum & Mean & S.D. \\
\hline Elderly female & 15 & 75.00 & 80.00 & 77.93 & 1.87 \\
\hline Elderly male & 15 & 75.00 & 80.00 & 78.80 & 1.57 \\
\hline patient female & 15 & 70.00 & 80.00 & 74.73 & 2.15 \\
\hline patient male & 15 & 70.00 & 80.00 & 74.67 & 2.16 \\
\hline Female Students College & 15 & 19.00 & 20.00 & 19.53 & 0.52 \\
\hline male Students College & 15 & 19.00 & 20.00 & 19.47 & 0.52 \\
\hline School Students Female & 15 & 9.00 & 12.00 & 10.67 & 1.11 \\
\hline School Students Male & 15 & 9.00 & 12.00 & 10.80 & 1.21 \\
\hline Female School students & 8 & 13.00 & 17.00 & 15.00 & 1.31 \\
\hline male School students & 7 & 14.00 & 17.00 & 16.00 & 1.15 \\
\hline swimming Female & 15 & 17.00 & 21.00 & 19.13 & 1.25 \\
\hline Swimming Male & 15 & 17.00 & 21.00 & 19.13 & 1.30 \\
\hline Female fencing & 15 & 17.00 & 21.00 & 19.27 & 1.39 \\
\hline male fencing & 15 & 17.00 & 21.00 & 19.33 & 1.50 \\
\hline female Athletics & 15 & 18.00 & 20.00 & 19.27 & 0.80 \\
\hline male Athletics & 15 & 18.00 & 21.00 & 19.53 & 1.13 \\
\hline Female Gymnastics & 15 & 7.00 & 10.00 & 8.93 & 0.88 \\
\hline male Gymnastics & 15 & 8.00 & 12.00 & 10.00 & 1.46 \\
\hline Female Taekwondo & 8 & 13.00 & 17.00 & 15.00 & 1.51 \\
\hline male Taekwondo & 7 & 14.00 & 17.00 & 15.29 & 1.11 \\
\hline Total & 270 & 7.00 & 80.00 & 35.82 & 22.16 \\
\hline ANOVA & & & & 116.5 & \\
P value & & & $0.0001^{*}$ & \\
\hline
\end{tabular}

Table (2): BMI distribution among the studied groups.

\begin{tabular}{l|c|c|c|c|c}
\hline \multicolumn{1}{c|}{ BMI } & N & Minimum & Maximum & Mean & S.D. \\
\hline Elderly female & 15 & 29.70 & 33.70 & 31.90 & 0.99 \\
\hline Elderly male & 15 & 30.50 & 34.10 & 32.04 & 0.95 \\
\hline Wheel chair female & 15 & 26.10 & 29.30 & 27.55 & 0.79 \\
\hline Wheel chair male & 15 & 26.90 & 30.10 & 28.39 & 0.80 \\
\hline University female Students & 15 & 21.00 & 24.50 & 22.99 & 1.05 \\
\hline University male Students & 15 & 19.80 & 22.50 & 21.56 & 0.70 \\
\hline Female 1ry School Students & 15 & 17.10 & 19.50 & 18.13 & 0.62 \\
\hline Male 1ry School Students & 15 & 16.80 & 18.40 & 17.70 & 0.47 \\
\hline Female 2ry School students & 8 & 21.70 & 25.90 & 24.58 & 1.28 \\
\hline Male 2ry School students & 7 & 21.20 & 22.90 & 21.73 & 0.59 \\
\hline Swimming Females & 15 & 21.20 & 23.00 & 21.96 & 0.45 \\
\hline Swimming Males & 15 & 18.00 & 18.80 & 18.49 & 0.27 \\
\hline Fencing Females & 15 & 21.30 & 23.80 & 21.94 & 0.60 \\
\hline Fencing Males & 15 & 21.80 & 23.00 & 22.41 & 0.34 \\
\hline Athletic Females & 15 & 20.60 & 22.10 & 21.24 & 0.44 \\
\hline Athletic Males & 15 & 21.50 & 23.30 & 22.45 & 0.45 \\
\hline Gymnastic Females & 15 & 16.50 & 19.20 & 17.86 & 0.86 \\
\hline Gymnastic Males & 15 & 17.80 & 20.40 & 19.09 & 0.84 \\
\hline Taekwondo Females & 8 & 24.10 & 25.90 & 24.91 & 0.64 \\
\hline Taekwondo Males & 7 & 20.80 & 21.70 & 21.24 & 0.29 \\
\hline Total & 270 & 16.50 & 34.10 & 23.31 & 3.60 \\
\hline ANOVA & & & 201 & \\
\hline P value & & & $0.0001 *$ & \\
\hline
\end{tabular}


Participants were required to have no upper or lower limb injury in the preceding two months that could potentially impact on the assessment of muscle force. This study used t-test while more than two populations were analyzed F-test (ANOVA), whereby participants attended two identical testing sessions. All participants gave written informed consent.

\section{Instrumentations:}

To determine BMI we used sensitive balance scale graduated tape. BMI is calculated by taking one's weight $(\mathrm{kg})$ and dividing the square of one's height $(\mathrm{m})$ [19].

The proposed device consists of two metal plates connected through a pivot that allowed both of them to move freely and independently Figure (1). Three parallel springs were placed in between the two plates to support the loading applied from the contraction of muscles as shown in Figure (2). The loading cause compression of the springs that will be in accordance to Hook's law and reliable correlation between loading and displacement could be maintained. The system of springs was connected to an electronic system to monitor the changes resulted from its compression, whereas any micro-changes in the spring length could be detected. Moreover, the body of the metal plates was also attached to resistive electronic sensor to reflect any angle deviations would be produced under the application of the loading. The obtained data were displayed on liquid crystal display (LCD) using custom-written Arduino software and soldered and compactly packed in a plastic casing $\left(20 \times 20 \mathrm{~cm}^{2}\right)$. For more applicability of the system of measurement, there were two separate antennas, one placed as transmitter from the body of the plates and the other was a wireless receiver at the plastic casing. An illustrative sketch of the main electronic components of the system is shown in Figure (3).

Furthermore, the system was calibrated under known weights to perform load-displacement curve of the system of springs. The calibration was attained by loading the system by weights of 1 $25 \mathrm{~kg}$ with increment of $1 \mathrm{~kg}$ as adapted elsewhere [10, 18]. It is worthy to mention that the calibration was repeated for each loading weight step 10 times and average values were taken for reliable measurements. Taking the advantage of the load-displacement curve to specify an equation to transform the deviation of the plates under loading conditions into force, one can directly convert the readings from the LCD screen into force.

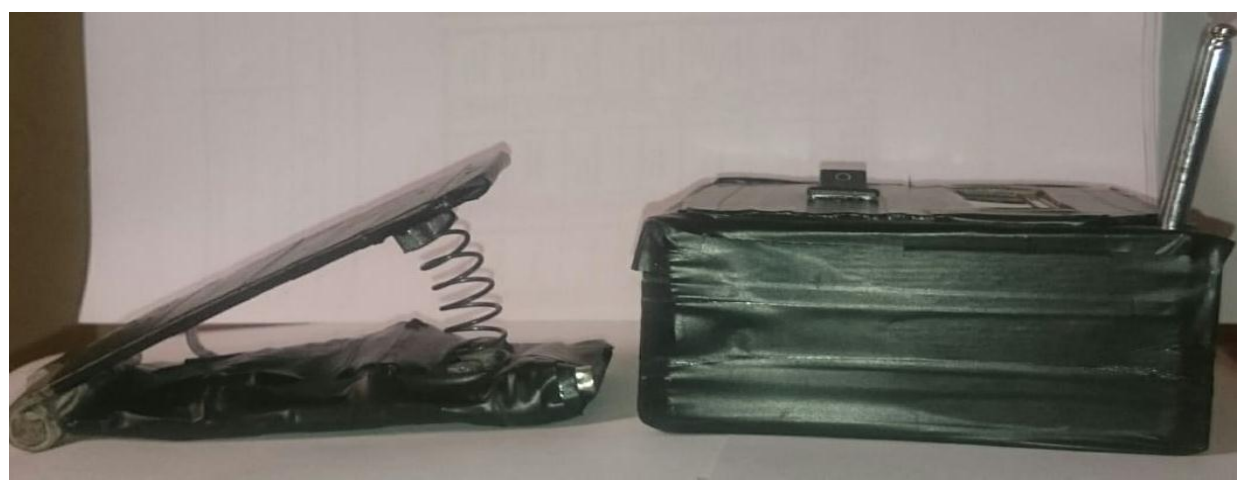

Figure 1: The device with its software.
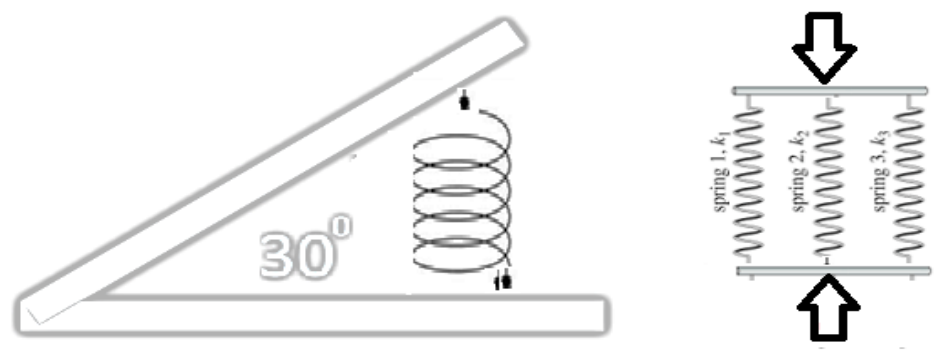

Figure 2: sketch diagram of the two metal plates sandwiching three springs in paralle 


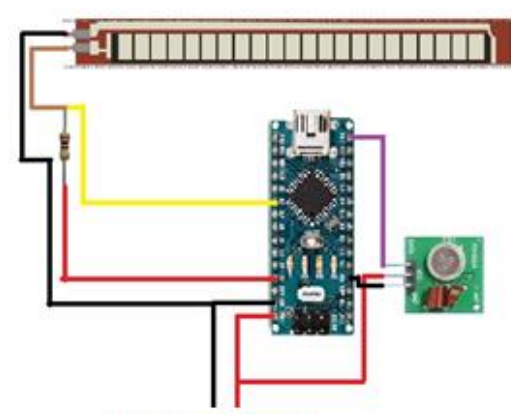

Transmitter

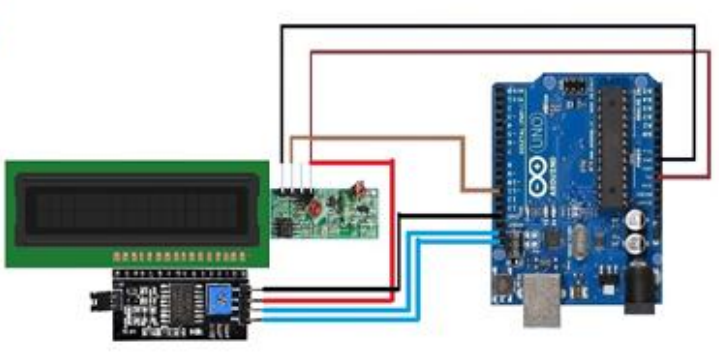

Receiver

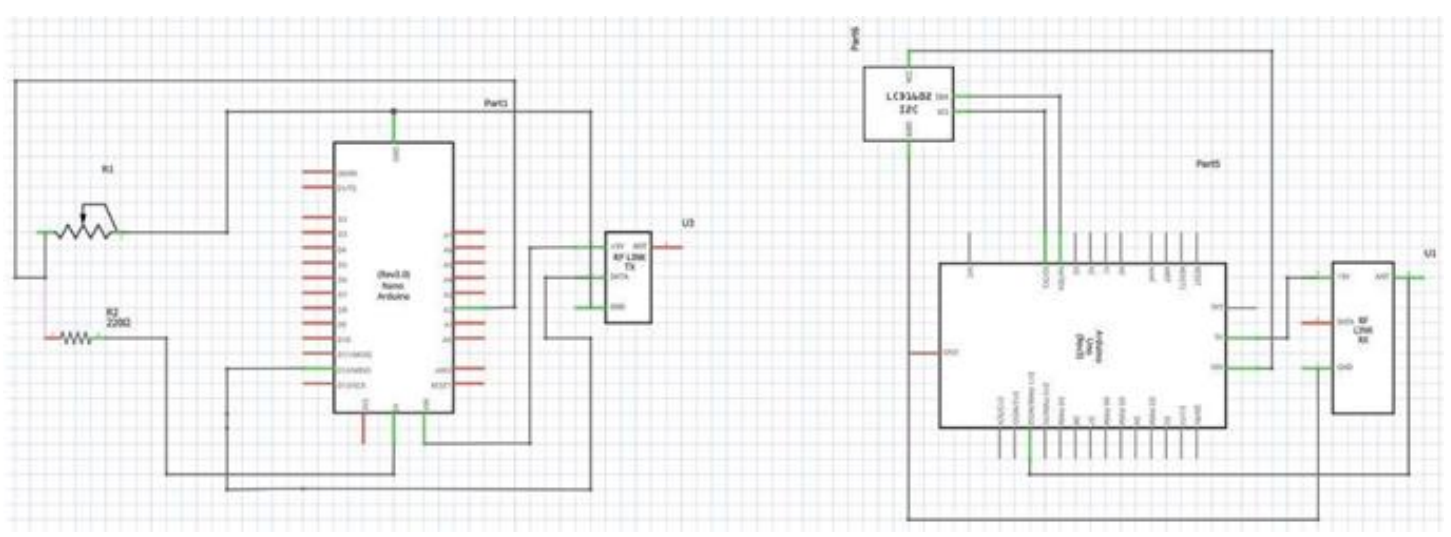

Figure 3: sketch diagram of the two electronic circuits for transmitting and receiving the data on the LCD screen.

\section{Device Calibration:}

The calibration of the device was maintained to perform load-displacement curve by applying known weights of $1-25 \mathrm{~kg}$ in step of $1 \mathrm{~kg}$. The readings displayed on the LCD screen were monitored and graphed versus the loading values and found to be linearly proportional at the range up to $17 \mathrm{~kg}$, after that nonlinear proportionality was obtained as shown in Figure (4). Therefore, the equation of calibration was taken from the linear part of the curve (Hook's law) and it was considered not to exceed these limits in the loading process during the measurements.

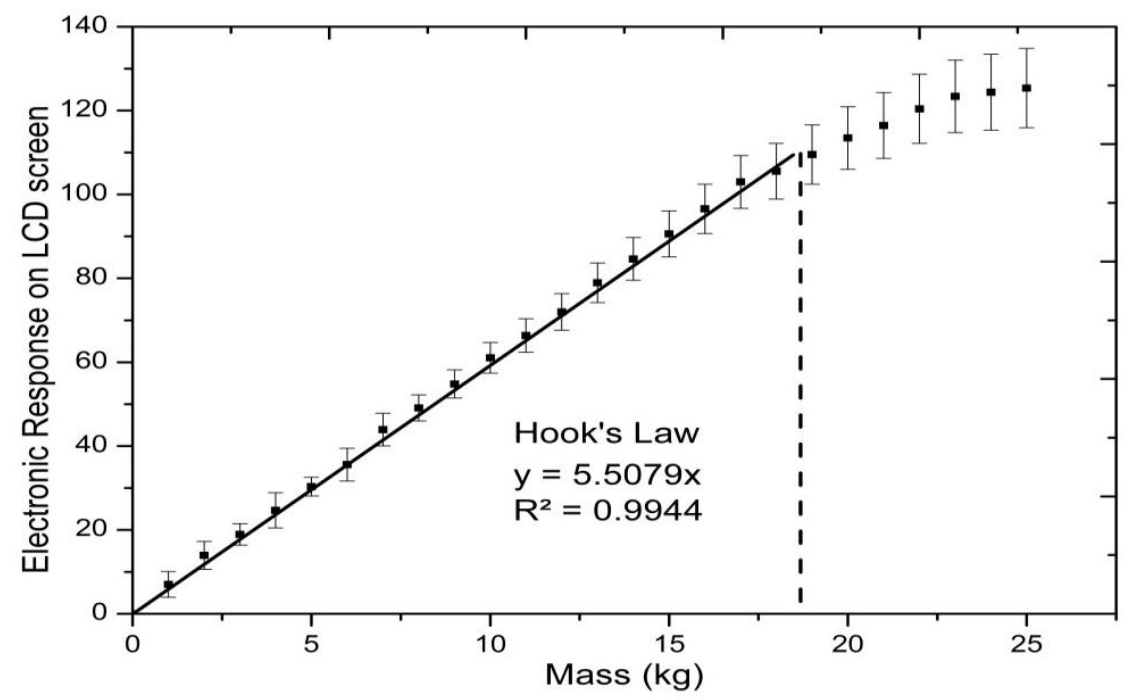

Figure 4: correlation between electronic response readings to the loading of masses and it represents the linear portion that obeying to Hook's law at loading up to $17 \mathrm{~kg}$. 
Procedure:

There are no literatures on the most appropriate testing positions for the device use for different groups of muscles. Based on prior research and our own pilot work of assessments in a variety of different positions, we implemented those shown in (figures: 5-8). These testing positions have shown strong reliability for the measurement of contractile force of different muscle groups.
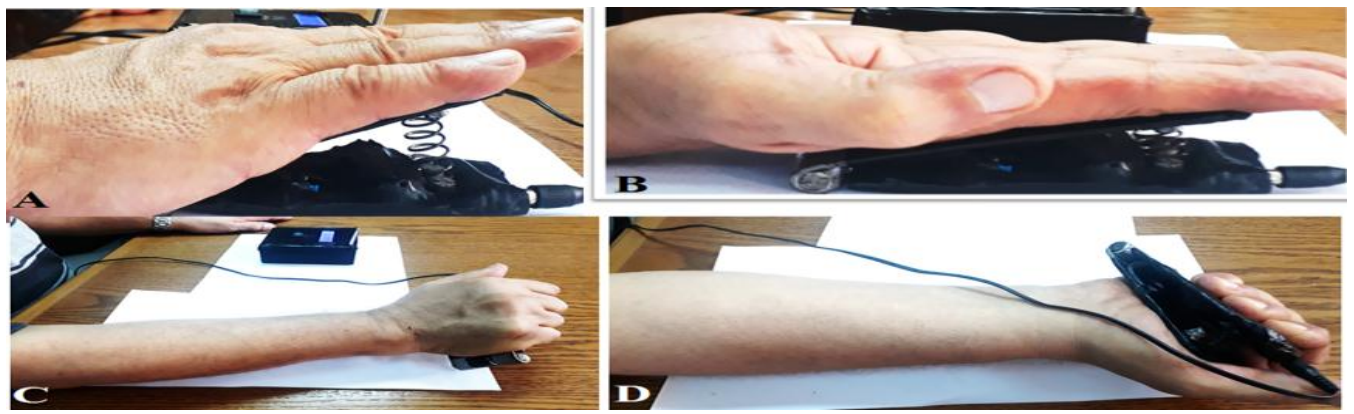

Figure 5: photograph showing different positions for testing different groups of muscles;
A: wrist flexors
B: wrist extensors
C: pronation
D: supination
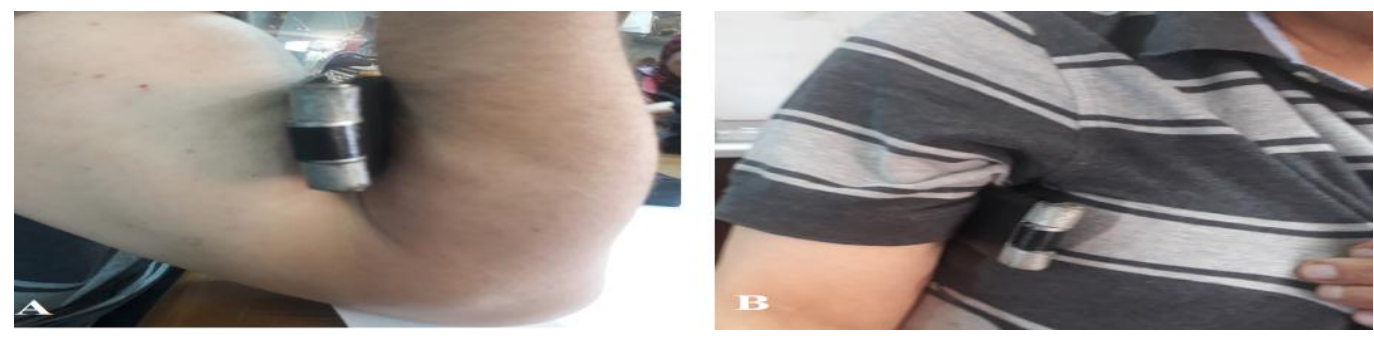

Figure 6: photograph showing different positions for testing different groups of muscles;

A: elbow flexors

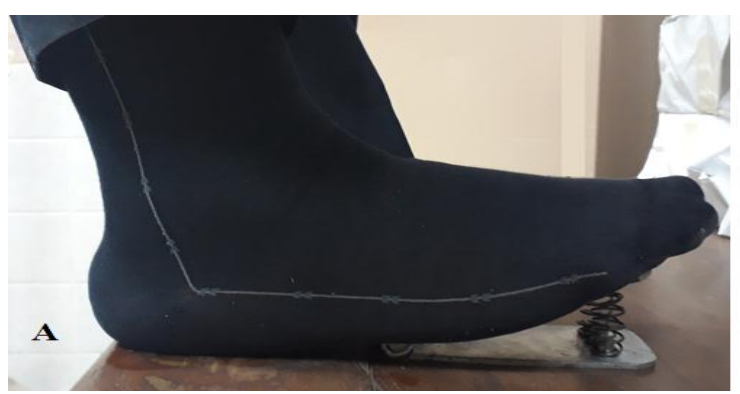

B: shoulder adductors

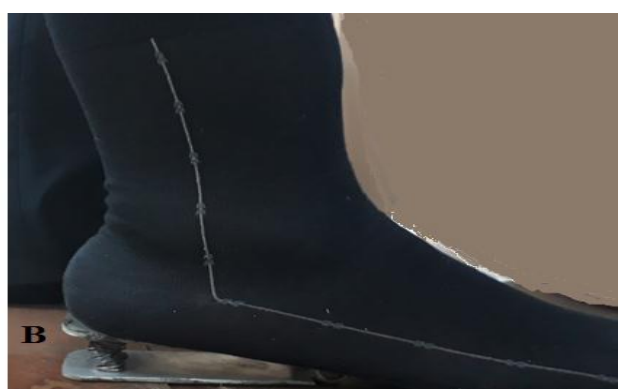

Figure 7: photograph showing different positions for testing different groups of muscles; A: plantarflexion of foot

B: dorsiflexion of foot
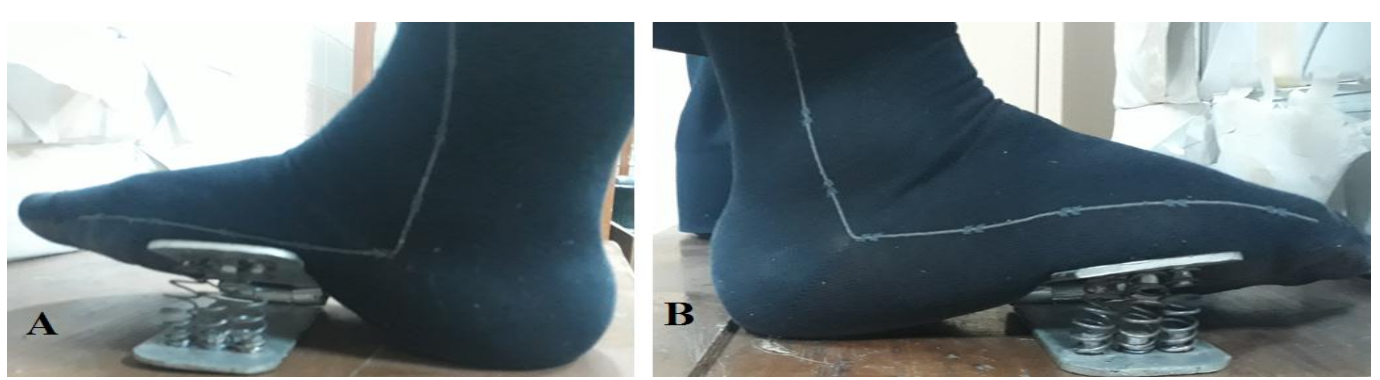

Figure 8: photograph showing different positions for testing different groups of muscles;

A: eversion of foot

B: inversion of foot 


\section{Statistical analysis of the data ${ }^{[6,8]}$}

Data were fed to the computer using IBM SPSS software package version 20.0. Quantitative data were described using mean and standard deviation for normally distributed data. For normally distributed data, comparison between two independent population were done using independent $\mathrm{t}$ test while more than two population were analyzed F-test (ANOVA) to be used. Significance test results are quoted as two-tailed probabilities. Significance of the obtained results was judged at the $5 \%$ level.

Where $\mathrm{X}$ and $\mathrm{Y}$ are the values of the first and second obs-ervations in the same individual. correlation can be significant $\mathrm{p}<0.05$.

Cohen's kappa coefficient $(\boldsymbol{\kappa})$ is a statistic which measures inter-rater agreement for qualitative (categorical) items. It is generally thought to be a more robust measure than simple percent agreement calculation, as $\kappa$ takes into account the possibility of the agreement occurring by chance. There is controversy surrounding Cohen's kappa due to the difficulty in interpreting indices of agreement. Some researchers have suggested that it is conceptually simpler to evaluate disagreement between items.

\section{Results:}

\section{Comparison between first and second measures for different groups of muscles:}

Comparison between measurements at first and second times for supination and pronation of upper limb, it was found that there was no significant difference between the 2 readings in both upper limbs, this data was shown in table (3).

On comparing measurements at first and second time for flexion and extension of the hand, it was found that there was no significant difference between the two readings regarding both upper limbs, this data was shown in table (3).

On comparing measurements at first and second time for flexion of the elbow, it was found that there was no significant difference between the two readings regarding both upper limbs, this data was shown in table (3).

On comparing measurements at first and second time for adduction of shoulder, it was found that there was no significant difference between the two readings regarding both upper limbs, this data was shown in table (3).

On comparing measurements at first and second time for eversion and inversion of the foot, it was found that there was no significant difference between the two readings regarding both lower limbs, this data was shown in table (4).

On comparing measurements at first and second time for planterflexion and dorsiflexion of the foot, it was found that there was no significant difference between the two readings regarding both lower limbs, this data was shown in table (4).

Table (5) shows the correlation between the 1 st and 2 nd measurements, from this table it was found that there was a highly significant positive correlation between the 1st and 2 nd measurements, the kappa in all variables was more than 0.70 , it indicates a high association and agreement of the two measurements. 
Table (3): Comparison between measurements at first and second time in all variables in all groups for upper limb.

\begin{tabular}{|c|c|c|c|c|c|c|c|c|c|c|c|c|}
\hline $\begin{array}{l}\text { Group } \\
\text { Mean } \pm \text { SD }\end{array}$ & $\begin{array}{c}\text { Pronation } \\
\text { Right } \\
\text { Forearm } \\
\end{array}$ & $\begin{array}{c}\begin{array}{c}\text { Pronation } \\
\text { Left }\end{array} \\
\text { Forearm } \\
\end{array}$ & $\begin{array}{l}\text { Supination } \\
\text { Right forarm }\end{array}$ & $\begin{array}{l}\text { Supination } \\
\text { left Forearm }\end{array}$ & $\begin{array}{c}\text { Flexion } \\
\text { Right Wrist }\end{array}$ & $\begin{array}{c}\text { Flexion Left } \\
\text { Wrist }\end{array}$ & $\begin{array}{l}\text { Extention } \\
\text { Right Wrist }\end{array}$ & $\begin{array}{l}\text { Extention } \\
\text { Left Wrist }\end{array}$ & $\begin{array}{c}\text { Flexion } \\
\text { Right Elbow }\end{array}$ & \begin{tabular}{|c} 
Flexion Left \\
Elbow
\end{tabular} & $\begin{array}{c}\text { Right } \\
\text { Shoulder }\end{array}$ & $\begin{array}{c}\text { Left } \\
\text { Shoulder }\end{array}$ \\
\hline $\begin{array}{l}\text { Elderly } \\
\text { female } 1^{\text {st }}\end{array}$ & $\begin{array}{c}2935.64 \pm 137 \\
.50\end{array}$ & $\begin{array}{c}28120.69 \pm 27 \\
1.08\end{array}$ & $\begin{array}{c}28739.31 \pm 22 \\
8.98\end{array}$ & $\begin{array}{c}28559.71 \pm 25 \\
6.38\end{array}$ & $\begin{array}{c}28992.08 \pm 23 \\
3.90\end{array}$ & $\begin{array}{c}28825.78 \pm 26 \\
1.42\end{array}$ & $\begin{array}{c}29058.60 \pm 19 \\
4.01\end{array}$ & $\begin{array}{c}28869.02 \pm 20 \\
7.99\end{array}$ & $\begin{array}{c}28310.26 \pm 25 \\
9.82\end{array}$ & $\begin{array}{c}28133.99 \pm 23 \\
2.83\end{array}$ & $\begin{array}{c}27771.47 \pm 22 \\
3.64\end{array}$ & $\begin{array}{c}27538.65 \pm 22 \\
3.11\end{array}$ \\
\hline $\begin{array}{l}\text { Elderly } \\
\text { female } 2^{\text {nd }}\end{array}$ & $\begin{array}{c}2935.63 \pm 137 \\
.49 \\
\end{array}$ & $\begin{array}{c}28120.69 \pm 27 \\
1.07 \\
\end{array}$ & \begin{tabular}{|c|}
$28739.31 \pm 22$ \\
8.97 \\
\end{tabular} & \begin{tabular}{|c|}
$28559.71 \pm 25$ \\
6.37 \\
\end{tabular} & $\begin{array}{c}28992.07 \pm 23 \\
3.89 \\
\end{array}$ & $\begin{array}{c}28825.78 \pm 26 \\
1.42 \\
\end{array}$ & $\begin{array}{c}29058.60 \pm 19 \\
4.02 \\
\end{array}$ & \begin{tabular}{|c|}
$28869.01 \pm 20$ \\
7.99 \\
\end{tabular} & \begin{tabular}{|c|}
$28310.27 \pm 25$ \\
9.83 \\
\end{tabular} & \begin{tabular}{|c|}
$28133.99 \pm 23$ \\
2.82 \\
\end{tabular} & $\begin{array}{c}27771.47 \pm 22 \\
3.63 \\
\end{array}$ & $\begin{array}{c}27538.65 \pm 22 \\
3.11 \\
\end{array}$ \\
\hline$P$ & 0.40 & 0.33 & 0.69 & 0.76 & 0.48 & 0.39 & 0.52 & 0.29 & 0.84 & 0.67 & 0.86 & 0.29 \\
\hline $\begin{array}{l}\text { Elderly } \\
\text { female } 1^{\text {st }}\end{array}$ & $\begin{array}{c}2937.33 \pm 22 . \\
77\end{array}$ & $\begin{array}{c}2891.52 \pm 22 . \\
77\end{array}$ & $\begin{array}{c}28885.65 \pm 22 \\
3.11\end{array}$ & $\begin{array}{c}28789.20 \pm 22 \\
8.98\end{array}$ & $\begin{array}{c}29035.32 \pm 22 \\
3.11\end{array}$ & $\begin{array}{c}28789.20 \pm 22 \\
8.98\end{array}$ & $\begin{array}{c}29184.98 \pm 22 \\
3.11\end{array}$ & $\begin{array}{c}29012.03 \pm 24 \\
5.76\end{array}$ & $\begin{array}{c}28935.54 \pm 22 \\
3.11\end{array}$ & $\begin{array}{c}28296.96 \pm 23 \\
7.92\end{array}$ & $\begin{array}{c}28792.52 \pm 23 \\
3.90\end{array}$ & $\begin{array}{c}28280.33 \pm 21 \\
4.88\end{array}$ \\
\hline $\begin{array}{l}\text { Elderly } \\
\text { female } 2^{\text {nd }}\end{array}$ & $\begin{array}{c}2937.34 \pm 22 . \\
76\end{array}$ & $\begin{array}{c}2891.53 \pm 22 . \\
77\end{array}$ & \begin{tabular}{|c|}
$28885.65 \pm 22$ \\
3.11
\end{tabular} & \begin{tabular}{|c|}
$28789.19 \pm 22$ \\
8.98
\end{tabular} & $\begin{array}{c}29035.31 \pm 22 \\
3.10\end{array}$ & $\begin{array}{c}28789.19 \pm 22 \\
8.98\end{array}$ & $\begin{array}{c}29184.97 \pm 22 \\
3.11\end{array}$ & $\begin{array}{c}29012.03 \pm 24 \\
5.75\end{array}$ & \begin{tabular}{|c|}
$28935.54 \pm 22$ \\
3.12 \\
\end{tabular} & \begin{tabular}{|c|}
$28296.97 \pm 23$ \\
7.91
\end{tabular} & $\begin{array}{c}28792.52 \pm 23 \\
3.90\end{array}$ & $\begin{array}{c}28280.33 \pm 21 \\
4.88\end{array}$ \\
\hline $\mathbf{P}$ & 0.40 & 0.33 & 0.69 & 0.76 & 0.48 & 0.39 & 0.52 & 0.29 & 0.84 & 0.67 & 0.86 & 0.29 \\
\hline $\begin{array}{l}\text { Patient } \\
\text { female1 }^{\text {st }}\end{array}$ & $\begin{array}{c}2876.25 \pm 22 . \\
77 \\
\end{array}$ & $\begin{array}{c}27788.10 \pm 22 \\
3.11 \\
\end{array}$ & \begin{tabular}{|c|}
$27987.65 \pm 22$ \\
3.11 \\
\end{tabular} & $\begin{array}{c}27754.84 \pm 24 \\
9.21 \\
\end{array}$ & $\begin{array}{c}28343.52 \pm 23 \\
3.90 \\
\end{array}$ & $\begin{array}{c}28207.16 \pm 24 \\
4.99 \\
\end{array}$ & $\begin{array}{c}28483.21 \pm 25 \\
5.41 \\
\end{array}$ & \begin{tabular}{|c|}
$28313.59 \pm 22$ \\
4.59 \\
\end{tabular} & \begin{tabular}{|c|}
$28306.94 \pm 24$ \\
4.99 \\
\end{tabular} & \begin{tabular}{|c|}
$28137.32 \pm 22$ \\
3.11 \\
\end{tabular} & $\begin{array}{c}27222.69 \pm 26 \\
5.78 \\
\end{array}$ & $\begin{array}{c}26956.61 \pm 29 \\
0.08 \\
\end{array}$ \\
\hline $\begin{array}{l}\text { Patient } \\
\text { female2 }^{\text {nd }}\end{array}$ & $\begin{array}{c}2876.25 \pm 22 . \\
76\end{array}$ & $\begin{array}{c}27788.10 \pm 22 \\
3.10\end{array}$ & $\begin{array}{c}27987.65 \pm 22 \\
3.11\end{array}$ & $\begin{array}{c}27754.84 \pm 24 \\
9.20\end{array}$ & $\begin{array}{c}28343.53 \pm 23 \\
3.90\end{array}$ & $\begin{array}{c}28207.17 \pm 24 \\
4.98\end{array}$ & $\begin{array}{c}28483.21 \pm 25 \\
5.41\end{array}$ & $\begin{array}{c}28313.60 \pm 22 \\
4.59\end{array}$ & \begin{tabular}{|c|}
$28306.94 \pm 24$ \\
4.98
\end{tabular} & $\begin{array}{c}28137.33 \pm 22 \\
3.11\end{array}$ & $\begin{array}{c}27222.69 \pm 26 \\
5.77\end{array}$ & $\begin{array}{c}26956.61 \pm 29 \\
0.08\end{array}$ \\
\hline $\bar{P}$ & 0.42 & 0.80 & 0.30 & 0.26 & 0.82 & 0.32 & 0.89 & 0.51 & 0.45 & 0.43 & 0.49 & 0.76 \\
\hline $\begin{array}{l}\begin{array}{l}\text { Patient } \\
\text { male1 }\end{array} \\
\end{array}$ & $\begin{array}{c}2886.43 \pm 22 . \\
93 \\
\end{array}$ & $\begin{array}{c}28190.53 \pm 22 \\
8.98 \\
\end{array}$ & \begin{tabular}{|c|}
$28286.98 \pm 22$ \\
3.11 \\
\end{tabular} & \begin{tabular}{|c|}
$28104.06 \pm 28$ \\
8.85 \\
\end{tabular} & $\begin{array}{c}28286.98 \pm 22 \\
3.11 \\
\end{array}$ & $\begin{array}{c}28137.32 \pm 23 \\
4.76 \\
\end{array}$ & $\begin{array}{c}28536.43 \pm 22 \\
3.11 \\
\end{array}$ & \begin{tabular}{|c|}
$28286.98 \pm 22$ \\
3.11 \\
\end{tabular} & \begin{tabular}{|c|}
$28569.69 \pm 20$ \\
3.67 \\
\end{tabular} & \begin{tabular}{|c|}
$28426.67 \pm 23$ \\
7.92 \\
\end{tabular} & $\begin{array}{c}27721.58 \pm 25 \\
6.24 \\
\end{array}$ & $\begin{array}{c}27551.96 \pm 28 \\
8.73 \\
\end{array}$ \\
\hline $\begin{array}{l}\text { Patient } \\
\text { male2 }\end{array}$ & $\begin{array}{c}2886.43 \pm 22 . \\
93\end{array}$ & $\begin{array}{c}28190.54 \pm 22 \\
8.98\end{array}$ & \begin{tabular}{|c|}
$28286.99 \pm 22$ \\
3.10 \\
\end{tabular} & \begin{tabular}{|c|}
$28104.07 \pm 28$ \\
8.85 \\
\end{tabular} & $\begin{array}{c}28286.99 \pm 22 \\
3.10\end{array}$ & $\begin{array}{c}28137.33 \pm 23 \\
4.76\end{array}$ & $\begin{array}{c}28536.43 \pm 22 \\
3.11\end{array}$ & \begin{tabular}{|c|}
$28286.99 \pm 22$ \\
3.10 \\
\end{tabular} & \begin{tabular}{|c|}
$28569.69 \pm 20$ \\
3.66 \\
\end{tabular} & \begin{tabular}{|c|}
$28426.67 \pm 23$ \\
7.93 \\
\end{tabular} & $\begin{array}{c}27721.59 \pm 25 \\
6.24\end{array}$ & $\begin{array}{c}27551.95 \pm 28 \\
8.74\end{array}$ \\
\hline $\mathbf{v}$ & 0.49 & 0.72 & 0.60 & 0.64 & 0.38 & 0.80 & 0.36 & 0.59 & 0.40 & 0.41 & 0.26 & 0.76 \\
\hline $\begin{array}{l}\text { Female } \\
\text { Students } \\
{\text { College } 1^{\text {st }}}^{\text {st }}\end{array}$ & $\begin{array}{c}2966.18 \pm 21 \\
66\end{array}$ & $\begin{array}{c}28812.48 \pm 25 \\
9.82\end{array}$ & \begin{tabular}{|c}
$29035.32 \pm 22$ \\
3.11
\end{tabular} & $\begin{array}{c}28915.58 \pm 24 \\
4.99\end{array}$ & $\begin{array}{c}28985.43 \pm 22 \\
3.11\end{array}$ & $\begin{array}{c}28789.20 \pm 22 \\
8.98\end{array}$ & $\begin{array}{c}29012.03 \pm 24 \\
5.76\end{array}$ & \begin{tabular}{|c|}
$28799.18 \pm 30$ \\
6.07
\end{tabular} & $\begin{array}{c}29135.09 \pm 22 \\
3.11\end{array}$ & $\begin{array}{c}28962.15 \pm 26 \\
2.55\end{array}$ & $\begin{array}{c}28586.32 \pm 22 \\
3.11\end{array}$ & $\begin{array}{c}28420.02 \pm 24 \\
3.43\end{array}$ \\
\hline $\begin{array}{l}\text { Female } \\
\text { Students } \\
\text { College2 }^{\text {nd }}\end{array}$ & $\begin{array}{c}2966.19 \pm 21 . \\
65\end{array}$ & $\begin{array}{c}28812.48 \pm 25 \\
9.83\end{array}$ & \begin{tabular}{|c}
$29035.31 \pm 22$ \\
3.10
\end{tabular} & \begin{tabular}{|c}
$28915.58 \pm 24$ \\
5.00
\end{tabular} & $\begin{array}{c}28985.43 \pm 22 \\
3.11\end{array}$ & $\begin{array}{c}28789.19 \pm 22 \\
8.98\end{array}$ & $\begin{array}{c}29012.03 \pm 24 \\
5.75\end{array}$ & $\begin{array}{c}28799.17 \pm 30 \\
6.06\end{array}$ & $\begin{array}{c}29135.09 \pm 22 \\
3.10\end{array}$ & $\begin{array}{c}28962.15 \pm 26 \\
2.54\end{array}$ & $\begin{array}{c}28586.32 \pm 22 \\
3.10\end{array}$ & $\begin{array}{c}28420.02 \pm 24 \\
3.44\end{array}$ \\
\hline $\mathbf{P}$ & 0.41 & 0.59 & 0.79 & 0.34 & 0.94 & 0.26 & 0.39 & 0.65 & 0.78 & 0.51 & 0.75 & 0.40 \\
\hline $\begin{array}{l}\text { Male } \\
\text { Students } \\
\text { College }^{\text {st }}\end{array}$ & $\begin{array}{c}2970.59 \pm 20 \\
53\end{array}$ & $\begin{array}{c}28935.54 \pm 22 \\
3.11\end{array}$ & \begin{tabular}{|c}
$29135.09 \pm 22$ \\
3.11
\end{tabular} & \begin{tabular}{|c|}
$28965.47 \pm 24$ \\
9.30
\end{tabular} & $\begin{array}{c}29131.77 \pm 22 \\
8.98\end{array}$ & $\begin{array}{c}28935.54 \pm 22 \\
3.11\end{array}$ & $\begin{array}{c}29135.09 \pm 30 \\
7.54\end{array}$ & \begin{tabular}{|c|}
$28822.46 \pm 24$ \\
1.08
\end{tabular} & \begin{tabular}{|c}
$29334.65 \pm 22$ \\
3.11
\end{tabular} & $\begin{array}{c}28982.10 \pm 28 \\
2.51\end{array}$ & $\begin{array}{c}28573.01 \pm 24 \\
1.08\end{array}$ & $\begin{array}{c}28400.06 \pm 22 \\
2.68\end{array}$ \\
\hline $\begin{array}{l}\text { Male } \\
\text { Students } \\
\text { College2 }^{\text {nd }}\end{array}$ & $\begin{array}{c}2970.59 \pm 20 \\
53\end{array}$ & $\begin{array}{c}28935.54 \pm 22 \\
3.12\end{array}$ & $\begin{array}{c}29135.09 \pm 22 \\
3.10\end{array}$ & $\begin{array}{c}28965.47 \pm 24 \\
9.31\end{array}$ & $\begin{array}{c}29131.76 \pm 22 \\
8.98\end{array}$ & $\begin{array}{c}28935.54 \pm 22 \\
3.12\end{array}$ & $\begin{array}{c}29135.09 \pm 30 \\
7.53\end{array}$ & \begin{tabular}{|c}
$28822.45 \pm 24$ \\
1.08
\end{tabular} & \begin{tabular}{|c}
$29334.65 \pm 22$ \\
3.11
\end{tabular} & $\begin{array}{c}28982.09 \pm 28 \\
2.51\end{array}$ & $\begin{array}{c}28573.01 \pm 24 \\
1.08\end{array}$ & $\begin{array}{c}28400.07 \pm 22 \\
2.69\end{array}$ \\
\hline $\mathbf{P}$ & 0.30 & 0.88 & 0.88 & 0.33 & 0.77 & 0.23 & 0.33 & 0.46 & 0.22 & 0.58 & 0.25 & 0.82 \\
\hline $\begin{array}{l}\text { School } \\
\text { Students } \\
\text { Female1 }^{\text {st }}\end{array}$ & $\begin{array}{c}2911.88 \pm 22 . \\
76\end{array}$ & $\begin{array}{c}28366.81 \pm 24 \\
4.99\end{array}$ & \begin{tabular}{|c}
$28489.86 \pm 24$ \\
8.35
\end{tabular} & \begin{tabular}{|c}
$28330.22 \pm 22$ \\
5.38
\end{tabular} & $\begin{array}{c}28286.98 \pm 22 \\
3.11\end{array}$ & $\begin{array}{c}28153.95 \pm 24 \\
3.43\end{array}$ & $\begin{array}{c}28373.46 \pm 24 \\
1.08\end{array}$ & \begin{tabular}{|c}
$28240.42 \pm 22$ \\
1.89
\end{tabular} & \begin{tabular}{|c}
$28127.34 \pm 22$ \\
8.77
\end{tabular} & \begin{tabular}{|c}
$27961.04 \pm 25$ \\
7.07
\end{tabular} & $\begin{array}{c}28882.32 \pm 22 \\
8.98\end{array}$ & $\begin{array}{c}28742.64 \pm 23 \\
3.90\end{array}$ \\
\hline $\begin{array}{l}\text { School } \\
\text { Students } \\
\text { Female2 }^{\text {nd }}\end{array}$ & $\begin{array}{c}2911.87 \pm 22 . \\
77\end{array}$ & $\begin{array}{c}28366.81 \pm 24 \\
4.99\end{array}$ & \begin{tabular}{|c}
$28489.87 \pm 24$ \\
8.35
\end{tabular} & $\begin{array}{c}28330.22 \pm 22 \\
5.38\end{array}$ & $\begin{array}{c}28286.99 \pm 22 \\
3.10\end{array}$ & $\begin{array}{c}28153.95 \pm 24 \\
3.43\end{array}$ & $\begin{array}{c}28373.46 \pm 24 \\
1.08\end{array}$ & \begin{tabular}{|c}
$28240.43 \pm 22$ \\
1.88
\end{tabular} & $\begin{array}{c}28127.35 \pm 22 \\
8.79\end{array}$ & $\begin{array}{c}27961.05 \pm 25 \\
7.07\end{array}$ & $\begin{array}{c}28882.32 \pm 22 \\
8.99\end{array}$ & $\begin{array}{c}28742.63 \pm 23 \\
3.89\end{array}$ \\
\hline $\bar{P}$ & 0.30 & 0.88 & 0.88 & 0.33 & 0.77 & 0.23 & 0.33 & 0.46 & 0.22 & 0.58 & 0.25 & 0.82 \\
\hline $\begin{array}{l}\text { School } \\
\text { Students } \\
\text { Male1 }^{\text {st }}\end{array}$ & $\begin{array}{c}2937.33 \pm 22 . \\
77\end{array}$ & $\begin{array}{c}28646.18 \pm 23 \\
7.92\end{array}$ & \begin{tabular}{|c}
$28825.78 \pm 23$ \\
7.92
\end{tabular} & $\begin{array}{c}28596.29 \pm 23 \\
7.92\end{array}$ & $\begin{array}{c}28745.96 \pm 23 \\
7.92\end{array}$ & $\begin{array}{c}28602.95 \pm 28 \\
2.00\end{array}$ & $\begin{array}{c}28606.27 \pm 25 \\
6.33\end{array}$ & \begin{tabular}{|c|}
$28446.63 \pm 26$ \\
3.45
\end{tabular} & $\begin{array}{c}28905.61 \pm 20 \\
2.03\end{array}$ & $\begin{array}{c}28656.16 \pm 21 \\
4.83\end{array}$ & $\begin{array}{c}28978.78 \pm 23 \\
5.41\end{array}$ & $\begin{array}{c}28839.09 \pm 24 \\
8.35\end{array}$ \\
\hline
\end{tabular}




\begin{tabular}{|c|c|c|c|c|c|c|c|c|c|c|c|c|}
\hline $\begin{array}{l}\text { Group } \\
\text { Mean } \pm S D\end{array}$ & $\begin{array}{c}\text { Pronation } \\
\text { Right } \\
\text { Forearm } \\
\end{array}$ & 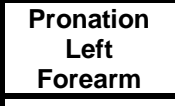 & $\begin{array}{l}\text { Supination } \\
\text { Right forarm }\end{array}$ & $\begin{array}{l}\text { Supination } \\
\text { left Forearm }\end{array}$ & $\begin{array}{c}\text { Flexion } \\
\text { Right Wrist }\end{array}$ & \begin{tabular}{|c} 
Flexion Left \\
Wrist
\end{tabular} & \begin{tabular}{|l} 
Extention \\
Right Wrist
\end{tabular} & $\begin{array}{l}\text { Extention } \\
\text { Left Wrist }\end{array}$ & $\begin{array}{c}\text { Flexion } \\
\text { Right Elbow }\end{array}$ & $\begin{array}{l}\text { Flexion Left } \\
\text { Elbow }\end{array}$ & $\begin{array}{l}\text { Right } \\
\text { Shoulder }\end{array}$ & $\begin{array}{l}\text { Left } \\
\text { Shoulder }\end{array}$ \\
\hline $\begin{array}{l}\text { School } \\
\text { Students } \\
\text { Male2 }\end{array}$ & \begin{tabular}{|c}
$2937.34 \pm 22$. \\
76
\end{tabular} & \begin{tabular}{|c}
$28646.19 \pm 23$ \\
7.91
\end{tabular} & \begin{tabular}{|c}
$28825.78 \pm 23$ \\
7.91
\end{tabular} & $\begin{array}{c}28596.29 \pm 23 \\
7.90\end{array}$ & $\begin{array}{c}28745.96 \pm 23 \\
7.91\end{array}$ & \begin{tabular}{|c|}
$28602.95 \pm 28$ \\
1.99
\end{tabular} & \begin{tabular}{|c|}
$28606.27 \pm 25$ \\
6.32
\end{tabular} & \begin{tabular}{|c|}
$28446.63 \pm 26$ \\
3.46
\end{tabular} & $\begin{array}{c}28905.61 \pm 20 \\
2.04\end{array}$ & $\begin{array}{c}28656.16 \pm 21 \\
4.82\end{array}$ & $\begin{array}{c}28978.77 \pm 23 \\
5.42\end{array}$ & $\begin{array}{c}28839.09 \pm 24 \\
8.35\end{array}$ \\
\hline $\bar{P}$ & 0.67 & 0.50 & 0.42 & 0.40 & 0.46 & 0.58 & 0.67 & 0.30 & 0.89 & 0.75 & 0.27 & 0.64 \\
\hline $\begin{array}{l}\text { Female } \\
\text { School } \\
\text { students } \\
\mathbf{1}^{\text {st }}\end{array}$ & $\begin{array}{c}9322.18 \pm 14 \\
0.48\end{array}$ & $\begin{array}{c}29191.22 \pm 18 \\
1.72\end{array}$ & $\begin{array}{c}29397.01 \pm 10 \\
5.83\end{array}$ & $\begin{array}{c}29241.11 \pm 11 \\
7.57\end{array}$ & $\begin{array}{c}28985.43 \pm 15 \\
5.49\end{array}$ & $\begin{array}{c}28866.94 \pm 14 \\
5.91\end{array}$ & $\begin{array}{c}28885.65 \pm 22 \\
1.51\end{array}$ & \begin{tabular}{|c}
$28723.51 \pm 23$ \\
2.09
\end{tabular} & $\begin{array}{c}28979.19 \pm 18 \\
1.72\end{array}$ & $\begin{array}{c}28823.29 \pm 15 \\
7.20\end{array}$ & $\begin{array}{c}28985.43 \pm 20 \\
9.97\end{array}$ & $\begin{array}{c}28835.76 \pm 22 \\
1.51\end{array}$ \\
\hline $\begin{array}{l}\text { Female } \\
\text { School } \\
\text { students } \\
2^{\text {nd }}\end{array}$ & $\begin{array}{c}29322.18 \pm 14 \\
0.46\end{array}$ & $\begin{array}{c}29191.21 \pm 18 \\
1.72\end{array}$ & $\begin{array}{c}29397.00 \pm 10 \\
5.82\end{array}$ & $\begin{array}{c}29241.10 \pm 11 \\
7.55\end{array}$ & $\begin{array}{c}28985.43 \pm 15 \\
5.49\end{array}$ & $\begin{array}{c}28866.95 \pm 14 \\
5.90\end{array}$ & $\begin{array}{c}28885.65 \pm 22 \\
1.51\end{array}$ & $\begin{array}{c}28723.51 \pm 23 \\
2.09\end{array}$ & $\begin{array}{c}28979.20 \pm 18 \\
1.72\end{array}$ & $\begin{array}{c}28823.28 \pm 15 \\
7.19\end{array}$ & $\begin{array}{c}28985.44 \pm 20 \\
9.97\end{array}$ & $\begin{array}{c}28835.74 \pm 22 \\
1.51\end{array}$ \\
\hline $\mathrm{v}$ & 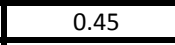 & 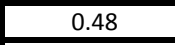 & 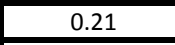 & - & $\cdots$ & 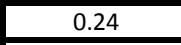 & $\cdots$ & 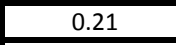 & 0.71 & .71 & 0.27 & 0.58 \\
\hline $\begin{array}{l}\text { Male } \\
\text { School } \\
\text { students } 1^{\text {s }} \\
t\end{array}$ & $\begin{array}{c}2988.97 \pm 12 \\
27\end{array}$ & $\begin{array}{c}2975.15 \pm 14 . \\
35\end{array}$ & \begin{tabular}{|c}
$29284.76 \pm 10$ \\
7.77
\end{tabular} & $\begin{array}{c}29135.09 \pm 10 \\
7.77\end{array}$ & $\begin{array}{c}29099.46 \pm 13 \\
4.22\end{array}$ & $\begin{array}{c}28999.68 \pm 13 \\
4.22\end{array}$ & $\begin{array}{c}29013.94 \pm 17 \\
9.55\end{array}$ & \begin{tabular}{|c}
$28835.76 \pm 10$ \\
7.77
\end{tabular} & $\begin{array}{c}28800.13 \pm 30 \\
7.15\end{array}$ & $\begin{array}{c}28636.21 \pm 29 \\
6.55\end{array}$ & $\begin{array}{c}28686.10 \pm 26 \\
3.99\end{array}$ & $\begin{array}{c}28522.17 \pm 34 \\
2.89\end{array}$ \\
\hline $\begin{array}{l}\text { Male } \\
\text { School } \\
\text { students2 } \\
\text { d }\end{array}$ & $\begin{array}{c}2988.97 \pm 12 \\
25\end{array}$ & $\begin{array}{c}2975.16 \pm 14 \\
34\end{array}$ & $\begin{array}{c}29284.76 \pm 10 \\
7.75\end{array}$ & $\begin{array}{c}29135.10 \pm 10 \\
7.80\end{array}$ & $\begin{array}{c}29099.44 \pm 13 \\
4.22\end{array}$ & $\begin{array}{c}28999.67 \pm 13 \\
4.23\end{array}$ & $\begin{array}{c}29013.91 \pm 17 \\
9.54\end{array}$ & $\begin{array}{c}28835.76 \pm 10 \\
7.75\end{array}$ & $\begin{array}{c}28800.11 \pm 30 \\
7.17\end{array}$ & $\begin{array}{c}28636.21 \pm 29 \\
6.53\end{array}$ & $\begin{array}{c}28686.09 \pm 26 \\
3.98\end{array}$ & $\begin{array}{c}28522.19 \pm 34 \\
2.87\end{array}$ \\
\hline $\mathbf{P}$ & 0.36 & 0.32 & 43 & 0.31 & 0.27 & 0.57 & 0.31 & 0.42 & 0.65 & 0.29 & 0.35 & 0.62 \\
\hline $\begin{array}{l}\text { Swimming } \\
\text { Female1 }\end{array}$ & \begin{tabular}{|c|}
$3008.60 \pm 22$ \\
77 \\
\end{tabular} & \begin{tabular}{|c|}
$29584.09 \pm 21$ \\
2.50 \\
\end{tabular} & \begin{tabular}{|c|}
$29444.41 \pm 24$ \\
6.72 \\
\end{tabular} & $\begin{array}{c}29574.12 \pm 22 \\
8.78 \\
\end{array}$ & \begin{tabular}{|c|}
$29334.65 \pm 23$ \\
7.02 \\
\end{tabular} & \begin{tabular}{|c|}
$29484.32 \pm 22$ \\
3.11 \\
\end{tabular} & \begin{tabular}{|c|}
$29311.37 \pm 24$ \\
5.76 \\
\end{tabular} & \begin{tabular}{|c|}
$29480.99 \pm 22$ \\
8.98 \\
\end{tabular} & $\begin{array}{c}29281.44 \pm 22 \\
0.28 \\
\end{array}$ & $\begin{array}{c}29510.92 \pm 22 \\
1.40 \\
\end{array}$ & $\begin{array}{c}28958.82 \pm 25 \\
8.45 \\
\end{array}$ & $\begin{array}{c}29474.34 \pm 23 \\
7.92 \\
\end{array}$ \\
\hline $\begin{array}{l}\text { Swimming } \\
\text { Female2 }^{\text {nd }} \\
\end{array}$ & \begin{tabular}{|c|}
$3008.61 \pm 22$. \\
76 \\
\end{tabular} & \begin{tabular}{|c|}
$29584.09 \pm 21$ \\
2.49 \\
\end{tabular} & \begin{tabular}{|c|}
$29444.40 \pm 24$ \\
6.72 \\
\end{tabular} & \begin{tabular}{|c|}
$29574.11 \pm 22$ \\
8.77 \\
\end{tabular} & \begin{tabular}{|c|}
$29334.64 \pm 23$ \\
7.03 \\
\end{tabular} & \begin{tabular}{|c|}
$29484.31 \pm 22$ \\
3.10 \\
\end{tabular} & \begin{tabular}{|c|}
$29311.36 \pm 24$ \\
5.77 \\
\end{tabular} & \begin{tabular}{|c|}
$29480.99 \pm 22$ \\
8.98 \\
\end{tabular} & $\begin{array}{c}29281.43 \pm 22 \\
0.28 \\
\end{array}$ & $\begin{array}{c}29510.92 \pm 22 \\
1.40 \\
\end{array}$ & \begin{tabular}{|c|}
$28958.81 \pm 25$ \\
8.44 \\
\end{tabular} & $\begin{array}{c}29474.33 \pm 23 \\
7.91 \\
\end{array}$ \\
\hline $\mathbf{P}$ & 0.45 & 0.48 & 0.21 & 0.66 & 0.86 & 0.24 & 0.53 & 0.21 & 0.71 & 0.71 & 0.27 & 0.58 \\
\hline $\begin{array}{l}\text { Swimming } \\
\text { Male } 1^{\text {st }}\end{array}$ & $\begin{array}{c}003.17 \pm 23 . \\
37\end{array}$ & \begin{tabular}{|c|}
$3013.02 \pm 23$ \\
87 \\
\end{tabular} & \begin{tabular}{|c|}
$29284.76 \pm 22$ \\
3.11 \\
\end{tabular} & $\begin{array}{c}29524.23 \pm 23 \\
7.92 \\
\end{array}$ & $\begin{array}{c}29391.19 \pm 29 \\
6.87 \\
\end{array}$ & \begin{tabular}{|c|}
$29514.25 \pm 24$ \\
9.30 \\
\end{tabular} & \begin{tabular}{|c|}
$29381.21 \pm 24$ \\
0.35 \\
\end{tabular} & \begin{tabular}{|c|}
$29534.21 \pm 22$ \\
3.11 \\
\end{tabular} & $\begin{array}{c}29284.76 \pm 22 \\
3.11 \\
\end{array}$ & $\begin{array}{c}29467.69 \pm 24 \\
3.43 \\
\end{array}$ & $\begin{array}{c}29135.09 \pm 23 \\
9.26 \\
\end{array}$ & $\begin{array}{c}29268.13 \pm 24 \\
3.43 \\
\end{array}$ \\
\hline $\begin{array}{l}\text { Swimming } \\
\text { Male } 2^{\text {nd }}\end{array}$ & \begin{tabular}{|c|}
$3003.18 \pm 23$ \\
36 \\
\end{tabular} & \begin{tabular}{|c|}
$3013.01 \pm 23$. \\
86 \\
\end{tabular} & \begin{tabular}{|c|}
$29284.75 \pm 22$ \\
3.11 \\
\end{tabular} & \begin{tabular}{|c|}
$29524.22 \pm 23$ \\
7.91 \\
\end{tabular} & \begin{tabular}{|c|}
$29391.18 \pm 29$ \\
6.87 \\
\end{tabular} & \begin{tabular}{|c|}
$29514.25 \pm 24$ \\
9.29 \\
\end{tabular} & \begin{tabular}{|c|}
$29381.21 \pm 24$ \\
0.36 \\
\end{tabular} & \begin{tabular}{|c|}
$29534.20 \pm 22$ \\
3.10 \\
\end{tabular} & $\begin{array}{c}29284.75 \pm 22 \\
3.11 \\
\end{array}$ & $\begin{array}{c}29467.69 \pm 24 \\
3.43 \\
\end{array}$ & \begin{tabular}{|c|}
$29135.09 \pm 23$ \\
9.26 \\
\end{tabular} & $\begin{array}{c}29268.12 \pm 24 \\
3.44 \\
\end{array}$ \\
\hline $\mathbf{P}$ & 0.52 & 0.53 & 0.29 & 0.49 & 0.85 & 0.77 & 0.80 & 0.84 & 0.76 & 0.84 & 0.88 & 0.31 \\
\hline $\begin{array}{l}\text { Female } \\
\text { fencing } 1^{\text {st }}\end{array}$ & $\begin{array}{c}010.64 \pm 25 . \\
00\end{array}$ & $\begin{array}{c}9414.47 \pm 24 \\
4.99 \\
\end{array}$ & \begin{tabular}{|c|}
$29500.95 \pm 24$ \\
3.43 \\
\end{tabular} & $\begin{array}{c}29391.19 \pm 27 \\
0.55 \\
\end{array}$ & \begin{tabular}{|c|}
$29487.64 \pm 28$ \\
1.88 \\
\end{tabular} & \begin{tabular}{|c|}
$29351.28 \pm 27$ \\
5.63 \\
\end{tabular} & \begin{tabular}{|c|}
$29384.54 \pm 22$ \\
3.11 \\
\end{tabular} & \begin{tabular}{|c|}
$29254.83 \pm 25$ \\
9.78 \\
\end{tabular} & $\begin{array}{c}29161.70 \pm 26 \\
2.55 \\
\end{array}$ & $\begin{array}{c}29022.01 \pm 24 \\
1.08 \\
\end{array}$ & $\begin{array}{c}29341.30 \pm 31 \\
6.57 \\
\end{array}$ & $\begin{array}{c}29045.29 \pm 27 \\
1.42 \\
\end{array}$ \\
\hline $\begin{array}{l}\text { Female } \\
\text { fencing2 }^{\text {nd }}\end{array}$ & \begin{tabular}{|c|}
$3010.64 \pm 24$. \\
99 \\
\end{tabular} & \begin{tabular}{|c|}
$29414.47 \pm 24$ \\
4.99 \\
\end{tabular} & \begin{tabular}{|c|}
$29500.94 \pm 24$ \\
3.42 \\
\end{tabular} & $\begin{array}{c}29391.19 \pm 27 \\
0.56 \\
\end{array}$ & \begin{tabular}{|c|}
$29487.63 \pm 28$ \\
1.88 \\
\end{tabular} & \begin{tabular}{|c|}
$29351.27 \pm 27$ \\
5.63 \\
\end{tabular} & \begin{tabular}{|c|}
$29384.54 \pm 22$ \\
3.12 \\
\end{tabular} & \begin{tabular}{|c|}
$29254.82 \pm 25$ \\
9.78 \\
\end{tabular} & $\begin{array}{c}29161.69 \pm 26 \\
2.54 \\
\end{array}$ & \begin{tabular}{|c|}
$29022.01 \pm 24$ \\
1.08 \\
\end{tabular} & $\begin{array}{c}29341.29 \pm 31 \\
6.59 \\
\end{array}$ & $\begin{array}{c}29045.29 \pm 27 \\
1.42 \\
\end{array}$ \\
\hline $\mathbf{P}$ & 0.31 & 0.49 & 0.60 & 0.25 & 0.94 & 0.21 & 0.78 & 0.64 & 0.89 & 0.62 & 0.57 & 0.63 \\
\hline $\begin{array}{l}\text { Male } \\
\text { fencing } 1^{\text {st }}\end{array}$ & $\begin{array}{c}3010.64 \pm 25 \\
00\end{array}$ & $\begin{array}{c}29354.61 \pm 24 \\
4.98\end{array}$ & $\begin{array}{c}29500.95 \pm 25 \\
0.63\end{array}$ & $\begin{array}{c}29351.28 \pm 22 \\
0.44\end{array}$ & $\begin{array}{c}29534.21 \pm 22 \\
3.11\end{array}$ & $\begin{array}{c}29354.61 \pm 27 \\
9.56\end{array}$ & $\begin{array}{c}29301.39 \pm 24 \\
3.43\end{array}$ & $\begin{array}{c}29151.72 \pm 25 \\
0.63\end{array}$ & $\begin{array}{c}29155.05 \pm 35 \\
7.67\end{array}$ & $\begin{array}{c}29065.25 \pm 28 \\
5.22 \\
\end{array}$ & $\begin{array}{c}29181.66 \pm 45 \\
3.12\end{array}$ & $\begin{array}{c}28965.47 \pm 36 \\
8.44\end{array}$ \\
\hline $\begin{array}{l}\text { Male } \\
\text { fencing } 2^{\text {nd }}\end{array}$ & \begin{tabular}{|c|}
$3010.64 \pm 24$. \\
99 \\
\end{tabular} & \begin{tabular}{|c|}
$29354.61 \pm 24$ \\
5.00 \\
\end{tabular} & \begin{tabular}{|c|}
$29500.94 \pm 25$ \\
0.62 \\
\end{tabular} & $\begin{array}{c}29351.28 \pm 22 \\
0.44 \\
\end{array}$ & \begin{tabular}{|c|}
$29534.20 \pm 22$ \\
3.10 \\
\end{tabular} & \begin{tabular}{|c|}
$29354.60 \pm 27$ \\
9.56 \\
\end{tabular} & \begin{tabular}{|c|}
$29301.39 \pm 24$ \\
3.44 \\
\end{tabular} & \begin{tabular}{|c|}
$29151.71 \pm 25$ \\
0.62 \\
\end{tabular} & $\begin{array}{c}29155.05 \pm 35 \\
7.68 \\
\end{array}$ & \begin{tabular}{|c|}
$29065.24 \pm 28$ \\
5.22 \\
\end{tabular} & \begin{tabular}{|c|}
$29181.65 \pm 45$ \\
3.13 \\
\end{tabular} & \begin{tabular}{|c|}
$28965.47 \pm 36$ \\
8.44 \\
\end{tabular} \\
\hline $\mathbf{P}$ & 0.25 & 0.83 & 0.40 & 0.72 & 0.70 & 0.45 & 0.49 & 0.51 & 0.53 & 0.80 & 0.81 & 0.47 \\
\hline $\begin{array}{l}\text { Female } \\
\text { Athletics } \\
1^{\text {st }}\end{array}$ & \begin{tabular}{|c}
$3009.62 \pm 24$ \\
28
\end{tabular} & \begin{tabular}{|c}
$29394.52 \pm 23$ \\
7.92
\end{tabular} & \begin{tabular}{|c}
$29534.21 \pm 22$ \\
3.11
\end{tabular} & $\begin{array}{c}29427.78 \pm 24 \\
5.76\end{array}$ & $\begin{array}{c}29288.09 \pm 28 \\
0.61\end{array}$ & $\begin{array}{c}29168.35 \pm 25 \\
0.63\end{array}$ & $\begin{array}{c}29218.24 \pm 29 \\
0.08\end{array}$ & $\begin{array}{c}29091.86 \pm 26 \\
1.19\end{array}$ & $\begin{array}{c}29331.32 \pm 22 \\
8.98\end{array}$ & $\begin{array}{c}29121.79 \pm 27 \\
8.07\end{array}$ & $\begin{array}{c}29457.71 \pm 25 \\
7.07\end{array}$ & $\begin{array}{c}29228.22 \pm 25 \\
0.06\end{array}$ \\
\hline
\end{tabular}




\begin{tabular}{|c|c|c|c|c|c|c|c|c|c|c|c|c|}
\hline $\begin{array}{l}\text { Group } \\
\text { Mean } \pm S D\end{array}$ & $\begin{array}{l}\text { Pronation } \\
\text { Right } \\
\text { Forearm } \\
\end{array}$ & $\begin{array}{c}\begin{array}{c}\text { Pronation } \\
\text { Left }\end{array} \\
\text { Forearm } \\
\end{array}$ & $\begin{array}{c}\text { Supination } \\
\text { Right forarm }\end{array}$ & $\begin{array}{l}\text { Supination } \\
\text { left Forearm }\end{array}$ & $\begin{array}{c}\text { Flexion } \\
\text { Right Wrist }\end{array}$ & $\begin{array}{c}\begin{array}{c}\text { Flexion Left } \\
\text { Wrist }\end{array} \\
\end{array}$ & $\begin{array}{l}\text { Extention } \\
\text { Right Wrist }\end{array}$ & $\begin{array}{l}\text { Extention } \\
\text { Left Wrist }\end{array}$ & $\begin{array}{c}\text { Flexion } \\
\text { Right Elbow }\end{array}$ & $\begin{array}{l}\text { Flexion Left } \\
\text { Elbow }\end{array}$ & $\begin{array}{l}\text { Right } \\
\text { Shoulder }\end{array}$ & $\begin{array}{c}\text { Left } \\
\text { Shoulder }\end{array}$ \\
\hline $\begin{array}{l}\text { Female } \\
\text { Athletics } \\
2^{\text {nd }}\end{array}$ & $\begin{array}{c}3009.62 \pm 24 \\
26\end{array}$ & $\begin{array}{c}29394.51 \pm 23 \\
7.92\end{array}$ & $\begin{array}{c}29534.20 \pm 22 \\
3.10\end{array}$ & $\begin{array}{c}29427.77 \pm 24 \\
5.76\end{array}$ & $\begin{array}{c}29288.08 \pm 28 \\
0.63\end{array}$ & $\begin{array}{c}29168.35 \pm 25 \\
0.63\end{array}$ & $\begin{array}{c}29218.23 \pm 29 \\
0.08\end{array}$ & $\begin{array}{c}29091.85 \pm 26 \\
1.18\end{array}$ & $\begin{array}{c}29331.32 \pm 22 \\
8.99\end{array}$ & $\begin{array}{c}29121.78 \pm 27 \\
8.07\end{array}$ & $\begin{array}{c}29457.71 \pm 25 \\
7.07\end{array}$ & $\begin{array}{c}29228.21 \pm 25 \\
0.06\end{array}$ \\
\hline $\mathbf{P}$ & 0.25 & 0.88 & 0.90 & 0.41 & 0.44 & 0.60 & 0.78 & 0.57 & 0.55 & 0.81 & 0.40 & 0.40 \\
\hline $\begin{array}{l}\text { Male } \\
\text { Athletics } \\
1^{\text {st }}\end{array}$ & $\begin{array}{c}3010.64 \pm 25 \\
00\end{array}$ & \begin{tabular}{|c}
$29384.54 \pm 22$ \\
3.11
\end{tabular} & \begin{tabular}{|c}
$29480.99 \pm 22$ \\
8.98
\end{tabular} & $\begin{array}{c}29288.09 \pm 22 \\
8.98\end{array}$ & $\begin{array}{c}29464.36 \pm 25 \\
5.64\end{array}$ & $\begin{array}{c}29264.81 \pm 30 \\
1.58\end{array}$ & $\begin{array}{c}29361.26 \pm 26 \\
3.90\end{array}$ & \begin{tabular}{|c|}
$29238.20 \pm 24$ \\
6.91
\end{tabular} & $\begin{array}{c}29361.26 \pm 30 \\
3.97\end{array}$ & $\begin{array}{c}29194.96 \pm 32 \\
6.71\end{array}$ & $\begin{array}{c}29281.44 \pm 35 \\
9.98\end{array}$ & $\begin{array}{c}29128.44 \pm 33 \\
6.71\end{array}$ \\
\hline $\begin{array}{l}\text { Male } \\
\text { Athletics } \\
2^{\text {nd }}\end{array}$ & $\begin{array}{c}3010.64 \pm 24 . \\
99\end{array}$ & $\begin{array}{c}29384.54 \pm 22 \\
3.12\end{array}$ & $\begin{array}{c}29480.99 \pm 22 \\
8.98\end{array}$ & $\begin{array}{c}29288.08 \pm 22 \\
8.99\end{array}$ & $\begin{array}{c}29464.35 \pm 25 \\
5.64\end{array}$ & $\begin{array}{c}29264.80 \pm 30 \\
1.59\end{array}$ & $\begin{array}{c}29361.25 \pm 26 \\
3.90\end{array}$ & $\begin{array}{c}29238.19 \pm 24 \\
6.91\end{array}$ & $\begin{array}{c}29361.25 \pm 30 \\
3.98\end{array}$ & $\begin{array}{c}29194.96 \pm 32 \\
6.71\end{array}$ & $\begin{array}{c}29281.43 \pm 35 \\
9.99\end{array}$ & $\begin{array}{c}29128.43 \pm 33 \\
6.71\end{array}$ \\
\hline$\overline{\mathbf{P}}$ & 0.31 & 0.49 & 0.60 & 0.25 & 0.94 & 0.21 & 0.78 & 0.64 & 0.89 & 0.62 & 0.57 & 0.63 \\
\hline $\begin{array}{l}\text { Female } \\
\text { Gymnastic } \\
s 1^{\text {st }}\end{array}$ & $\begin{array}{c}2886.43 \pm 22 . \\
77 \\
\end{array}$ & \begin{tabular}{|c|}
$28519.80 \pm 24$ \\
3.43
\end{tabular} & \begin{tabular}{|c|}
$28267.03 \pm 30$ \\
2.17 \\
\end{tabular} & $\begin{array}{c}28403.39 \pm 29 \\
4.94 \\
\end{array}$ & \begin{tabular}{|c|}
$27708.27 \pm 25$ \\
3.54 \\
\end{tabular} & $\begin{array}{c}27891.20 \pm 24 \\
9.78 \\
\end{array}$ & $\begin{array}{c}28007.61 \pm 24 \\
4.98 \\
\end{array}$ & \begin{tabular}{|c}
$28286.98 \pm 22$ \\
3.11 \\
\end{tabular} & $\begin{array}{c}27788.10 \pm 22 \\
3.11 \\
\end{array}$ & $\begin{array}{c}28037.54 \pm 23 \\
4.76 \\
\end{array}$ & \begin{tabular}{|c}
$28835.76 \pm 22$ \\
3.11 \\
\end{tabular} & \begin{tabular}{|c|}
$29035.32 \pm 22$ \\
3.11 \\
\end{tabular} \\
\hline $\begin{array}{l}\text { Female } \\
\text { Gymnastic } \\
\text { s2 }\end{array}$ & $\begin{array}{c}2887.11 \pm 25 . \\
29\end{array}$ & $\begin{array}{c}2910.52 \pm 26 \\
63\end{array}$ & $\begin{array}{c}28273.69 \pm 31 \\
4.09\end{array}$ & $\begin{array}{c}28413.37 \pm 31 \\
0.90\end{array}$ & \begin{tabular}{|c|}
$27708.27 \pm 25$ \\
3.53
\end{tabular} & $\begin{array}{c}27891.20 \pm 24 \\
9.78\end{array}$ & $\begin{array}{c}28010.94 \pm 25 \\
0.78\end{array}$ & \begin{tabular}{|c|}
$28290.31 \pm 22$ \\
8.98
\end{tabular} & $\begin{array}{c}27788.10 \pm 22 \\
3.10\end{array}$ & $\begin{array}{c}28037.55 \pm 23 \\
4.77\end{array}$ & \begin{tabular}{|c}
$28835.75 \pm 22$ \\
3.11
\end{tabular} & $\begin{array}{c}29035.31 \pm 22 \\
3.10\end{array}$ \\
\hline $\mathbf{P}$ & 0.46 & 0.58 & 0.62 & 0.22 & 0.65 & 0.63 & 0.67 & 0.65 & 0.69 & 0.69 & 0.60 & 0.54 \\
\hline $\begin{array}{l}\text { Male } \\
\text { Gymnastic } \\
s 1^{\text {st }}\end{array}$ & $\begin{array}{c}2942.42 \pm 22 . \\
77\end{array}$ & $\begin{array}{c}29035.32 \pm 22 \\
3.11\end{array}$ & $\begin{array}{c}28286.98 \pm 22 \\
3.11\end{array}$ & $\begin{array}{c}28566.36 \pm 25 \\
3.54 \\
\end{array}$ & \begin{tabular}{|c}
$28293.64 \pm 23$ \\
3.90 \\
\end{tabular} & $\begin{array}{c}28539.75 \pm 23 \\
8.86\end{array}$ & $\begin{array}{c}28193.86 \pm 23 \\
3.90\end{array}$ & \begin{tabular}{|c|}
$28533.10 \pm 22$ \\
8.98
\end{tabular} & $\begin{array}{c}28785.87 \pm 22 \\
3.11\end{array}$ & $\begin{array}{c}28978.78 \pm 21 \\
3.22 \\
\end{array}$ & $\begin{array}{c}28759.26 \pm 20 \\
8.16 \\
\end{array}$ & \begin{tabular}{|c}
$28952.17 \pm 24$ \\
7.06
\end{tabular} \\
\hline $\begin{array}{l}\text { Male } \\
\text { Gymnastic } \\
\text { s2 }\end{array}$ & $\begin{array}{c}2942.77 \pm 23 . \\
37\end{array}$ & \begin{tabular}{|c}
$29041.97 \pm 23$ \\
3.89
\end{tabular} & \begin{tabular}{|c}
$28293.64 \pm 23$ \\
5.41
\end{tabular} & $\begin{array}{c}28573.01 \pm 26 \\
3.63\end{array}$ & $\begin{array}{c}28293.64 \pm 23 \\
3.89\end{array}$ & $\begin{array}{c}28539.76 \pm 23 \\
8.86\end{array}$ & $\begin{array}{c}28193.87 \pm 23 \\
3.89\end{array}$ & \begin{tabular}{|c}
$28536.43 \pm 23$ \\
3.24
\end{tabular} & $\begin{array}{c}28785.87 \pm 22 \\
3.11\end{array}$ & $\begin{array}{c}28978.78 \pm 21 \\
3.23\end{array}$ & $\begin{array}{c}28769.24 \pm 22 \\
9.91\end{array}$ & $\begin{array}{c}28958.82 \pm 26 \\
0.51\end{array}$ \\
\hline $\mathbf{P}$ & 0.75 & 0.64 & 0.60 & 0.51 & 0.42 & 0.22 & 0.33 & 0.50 & 0.79 & 0.87 & 0.93 & 0.66 \\
\hline $\begin{array}{l}\text { Female } \\
\text { Taekwond } \\
\text { o1 } 1^{\text {st }}\end{array}$ & $\begin{array}{c}3025.79 \pm 13 . \\
59\end{array}$ & $\begin{array}{c}29546.68 \pm 14 \\
0.47\end{array}$ & \begin{tabular}{|c|}
$29746.23 \pm 10$ \\
5.83
\end{tabular} & $\begin{array}{c}29609.04 \pm 12 \\
2.20\end{array}$ & \begin{tabular}{|c}
$29297.23 \pm 14$ \\
5.45
\end{tabular} & $\begin{array}{c}29209.93 \pm 12 \\
2.20\end{array}$ & $\begin{array}{c}29166.28 \pm 15 \\
0.70\end{array}$ & \begin{tabular}{|c}
$29029.08 \pm 14$ \\
4.68
\end{tabular} & $\begin{array}{c}28617.50 \pm 14 \\
0.95\end{array}$ & $\begin{array}{c}28386.76 \pm 16 \\
4.38\end{array}$ & $\begin{array}{c}28629.97 \pm 15 \\
1.88\end{array}$ & $\begin{array}{c}28424.18 \pm 18 \\
6.19\end{array}$ \\
\hline $\begin{array}{l}\text { Female } \\
\text { Taekwond } \\
\text { o2 }\end{array}$ & $\begin{array}{c}3025.79 \pm 13 \\
58\end{array}$ & $\begin{array}{c}29546.68 \pm 14 \\
0.51\end{array}$ & $\begin{array}{c}29746.23 \pm 10 \\
5.81\end{array}$ & $\begin{array}{c}29609.04 \pm 12 \\
2.21\end{array}$ & $\begin{array}{c}29297.23 \pm 14 \\
5.44\end{array}$ & $\begin{array}{c}29209.93 \pm 12 \\
2.19\end{array}$ & $\begin{array}{c}29166.26 \pm 15 \\
0.70\end{array}$ & $\begin{array}{c}29029.07 \pm 14 \\
4.70\end{array}$ & $\begin{array}{c}28617.49 \pm 14 \\
0.94\end{array}$ & $\begin{array}{c}28386.78 \pm 16 \\
4.38\end{array}$ & $\begin{array}{c}28629.96 \pm 15 \\
1.87\end{array}$ & $\begin{array}{c}28424.20 \pm 18 \\
6.19\end{array}$ \\
\hline $\mathbf{P}$ & 0.47 & 0.91 & 0.64 & 0.33 & 0.74 & 0.79 & 0.36 & 0.83 & 0.51 & 0.71 & 0.28 & 0.65 \\
\hline $\begin{array}{l}\text { Male } \\
\text { Taekwond } \\
\text { o1 } 1^{\text {st }}\end{array}$ & $\begin{array}{c}3031.88 \pm 15 \\
23\end{array}$ & $\begin{array}{c}29569.84 \pm 13 \\
4.22\end{array}$ & $\begin{array}{c}29691.00 \pm 12 \\
0.25\end{array}$ & $\begin{array}{c}29576.97 \pm 16 \\
4.02\end{array}$ & $\begin{array}{c}29477.19 \pm 12 \\
0.25\end{array}$ & $\begin{array}{c}29377.41 \pm 12 \\
0.25\end{array}$ & $\begin{array}{c}29413.05 \pm 14 \\
0.69\end{array}$ & $\begin{array}{c}29242.00 \pm 12 \\
0.25\end{array}$ & $\begin{array}{c}28835.76 \pm 10 \\
7.77\end{array}$ & $\begin{array}{c}28707.48 \pm 14 \\
3.60\end{array}$ & $\begin{array}{c}28899.90 \pm 13 \\
4.22\end{array}$ & $\begin{array}{c}28686.09 \pm 10 \\
7.77\end{array}$ \\
\hline $\begin{array}{l}\text { Male } \\
\text { Taekwond } \\
\text { o2 }\end{array}$ & $\begin{array}{c}3031.87 \pm 15 \\
22\end{array}$ & $\begin{array}{c}29569.84 \pm 13 \\
4.25\end{array}$ & $\begin{array}{c}29691.00 \pm 12 \\
0.23\end{array}$ & $\begin{array}{c}29576.96 \pm 16 \\
4.03\end{array}$ & $\begin{array}{c}29477.19 \pm 12 \\
0.25\end{array}$ & $\begin{array}{c}29377.40 \pm 12 \\
0.23\end{array}$ & $\begin{array}{c}29413.04 \pm 14 \\
0.68\end{array}$ & $\begin{array}{c}29242.00 \pm 12 \\
0.23\end{array}$ & $\begin{array}{c}28835.76 \pm 10 \\
7.75\end{array}$ & $\begin{array}{c}28707.47 \pm 14 \\
3.62\end{array}$ & $\begin{array}{c}28899.90 \pm 13 \\
4.21\end{array}$ & $\begin{array}{c}28686.10 \pm 10 \\
7.80\end{array}$ \\
\hline$\overline{\mathbf{P}}$ & 0.46 & 0.58 & 0.62 & 0.22 & 0.65 & 0.63 & 0.67 & 0.65 & 0.69 & 0.69 & 0.60 & 0.54 \\
\hline
\end{tabular}


Table (4): Comparison between measurements at first and second time in all variables in all groups for lower limb.

\begin{tabular}{|c|c|c|c|c|c|c|c|c|}
\hline Group Mean $\pm S D$ & $\begin{array}{c}\text { Right Foot } \\
\text { planterflexion }\end{array}$ & $\begin{array}{c}\text { Left Foot } \\
\text { planterflexion }\end{array}$ & $\begin{array}{c}\text { Right Foot } \\
\text { dorsiflexion }\end{array}$ & $\begin{array}{c}\text { Left Foot } \\
\text { dorsiflexion }\end{array}$ & $\begin{array}{l}\text { Right Foot } \\
\text { Eversion }\end{array}$ & $\begin{array}{l}\text { Left Foot } \\
\text { Eversion }\end{array}$ & $\begin{array}{l}\text { Right Foot } \\
\text { Inversion }\end{array}$ & $\begin{array}{l}\text { Left Foot } \\
\text { Inversion }\end{array}$ \\
\hline Elderly female $1^{\text {st }}$ & $29035.32 \pm 223.11$ & $28862.37 \pm 292.04$ & $29022.01 \pm 241.08$ & $28779.22 \pm 316.57$ & $28692.75 \pm 233.90$ & $28559.71 \pm 287.13$ & $28785.87 \pm 223.11$ & $28599.62 \pm 304.32$ \\
\hline Elderly female $2^{\text {nd }}$ & $29035.31 \pm 223.10$ & $28862.37 \pm 292.05$ & $29022.01 \pm 241.08$ & $28779.22 \pm 316.57$ & $28692.75 \pm 233.89$ & $28559.71 \pm 287.12$ & $28785.87 \pm 223.11$ & $28599.62 \pm 304.31$ \\
\hline $\mathbf{P}$ & 0.52 & 0.34 & 0.83 & 0.53 & 0.43 & 0.59 & 0.60 & 0.21 \\
\hline Elderly female $1^{\text {st }}$ & $29035.32 \pm 223.11$ & $28789.20 \pm 221.88$ & $29284.76 \pm 223.11$ & $29091.86 \pm 233.90$ & $28985.43 \pm 223.11$ & $28822.46 \pm 272.90$ & $28785.87 \pm 223.11$ & $28632.88 \pm 228.98$ \\
\hline Elderly female $2^{\text {nd }}$ & $29035.31 \pm 223.10$ & $28789.19 \pm 221.89$ & $29284.75 \pm 223.11$ & $29091.85 \pm 233.89$ & $28985.43 \pm 223.11$ & $28822.46 \pm 272.90$ & $28785.87 \pm 223.11$ & $28632.88 \pm 228.97$ \\
\hline $\mathbf{P}$ & 0.52 & 0.34 & 0.83 & 0.53 & 0.43 & 0.59 & 0.60 & 0.21 \\
\hline Patient female1 $^{\text {st }}$ & $28326.89 \pm 237.92$ & $28210.49 \pm 244.31$ & $28436.65 \pm 223.11$ & $28277.01 \pm 211.83$ & $28366.81 \pm 202.03$ & $28267.03 \pm 217.30$ & $28366.81 \pm 202.03$ & $28233.77 \pm 224.28$ \\
\hline Patient female2 ${ }^{\text {nd }}$ & $28326.90 \pm 237.91$ & $28210.49 \pm 244.30$ & $28436.65 \pm 223.11$ & $28277.01 \pm 211.83$ & $28366.81 \pm 202.04$ & $28267.03 \pm 217.29$ & $28366.81 \pm 202.04$ & $28233.77 \pm 224.27$ \\
\hline $\mathbf{P}$ & 0.55 & 0.29 & 0.56 & 0.81 & 0.81 & 0.87 & 0.94 & 0.60 \\
\hline${\text { Patient male } 1^{\text {st }}}$ & $28479.89 \pm 214.88$ & $28366.81 \pm 244.99$ & $28376.78 \pm 237.92$ & $28253.72 \pm 260.37$ & $28536.43 \pm 223.11$ & $28420.02 \pm 242.70$ & $28286.98 \pm 223.11$ & $28137.32 \pm 223.11$ \\
\hline Patient male2 $^{\text {nd }}$ & $28479.89 \pm 214.89$ & $28366.81 \pm 244.99$ & $28376.79 \pm 237.92$ & $28253.73 \pm 260.36$ & $28536.43 \pm 223.11$ & $28420.02 \pm 242.71$ & $28286.99 \pm 223.10$ & $28137.33 \pm 223.11$ \\
\hline $\mathbf{v}$ & 0.52 & 0.29 & 0.79 & 0.37 & 0.64 & 0.75 & 0.55 & 0.73 \\
\hline $\begin{array}{l}\text { Female Students } \\
{\text { College } 1^{\text {st }}}\end{array}$ & $29118.46 \pm 203.67$ & $28985.43 \pm 223.11$ & $29055.27 \pm 196.68$ & $28908.93 \pm 250.77$ & $28805.83 \pm 252.14$ & $28699.40 \pm 241.09$ & $28885.65 \pm 223.11$ & $28735.98 \pm 223.11$ \\
\hline $\begin{array}{l}\text { Female Students } \\
\text { College2 }^{\text {nd }}\end{array}$ & $29118.46 \pm 203.67$ & $28985.43 \pm 223.11$ & $29055.27 \pm 196.68$ & $28908.93 \pm 250.77$ & $28805.83 \pm 252.14$ & $28699.40 \pm 241.07$ & $28885.65 \pm 223.11$ & $28735.98 \pm 223.10$ \\
\hline $\mathbf{P}$ & 0.85 & 0.41 & 0.55 & 0.26 & 0.93 & 0.61 & 0.47 & 0.78 \\
\hline $\begin{array}{l}\text { Male Students } \\
\text { College }^{\text {st }}\end{array}$ & $29314.69 \pm 202.03$ & $29188.31 \pm 228.98$ & $29058.60 \pm 201.21$ & $28958.82 \pm 208.16$ & $29065.25 \pm 202.03$ & $28885.65 \pm 223.11$ & $28872.35 \pm 241.08$ & $28742.64 \pm 233.90$ \\
\hline $\begin{array}{l}\text { Male Students } \\
\text { College2 }^{\text {nd }}\end{array}$ & $29314.69 \pm 202.04$ & $29188.30 \pm 228.98$ & $29058.59 \pm 201.21$ & $28958.81 \pm 208.16$ & $29065.25 \pm 202.03$ & $28885.65 \pm 223.11$ & $28872.34 \pm 241.09$ & $28742.63 \pm 233.89$ \\
\hline $\mathbf{P}$ & 0.39 & 0.55 & 0.45 & 0.67 & 0.24 & 0.27 & 0.39 & 0.69 \\
\hline $\begin{array}{l}\text { School Students } \\
\text { Female1 }^{\text {st }}\end{array}$ & $28895.63 \pm 277.26$ & $28769.24 \pm 305.60$ & $28985.43 \pm 223.11$ & $28832.44 \pm 224.27$ & $28785.87 \pm 223.11$ & $28632.88 \pm 228.98$ & $29028.66 \pm 233.90$ & $28882.32 \pm 228.98$ \\
\hline $\begin{array}{l}\text { School Students } \\
\text { Female2 }^{\text {nd }}\end{array}$ & $28895.63 \pm 277.26$ & $28769.25 \pm 305.59$ & $28985.43 \pm 223.11$ & $28832.43 \pm 224.28$ & $28785.87 \pm 223.11$ & $28632.88 \pm 228.97$ & $29028.66 \pm 233.89$ & $28882.32 \pm 228.99$ \\
\hline $\mathbf{P}$ & 0.39 & 0.55 & 0.45 & 0.67 & 0.24 & 0.27 & 0.39 & 0.69 \\
\hline $\begin{array}{l}\text { School Students } \\
\text { Male1 }^{\text {st }}\end{array}$ & $29035.32 \pm 223.11$ & $28918.91 \pm 243.43$ & $29085.21 \pm 223.11$ & $28885.65 \pm 223.11$ & $29131.77 \pm 228.98$ & $29008.71 \pm 245.76$ & $29035.32 \pm 223.11$ & $28859.04 \pm 259.82$ \\
\hline $\begin{array}{l}\text { School Students } \\
\text { Male2 }\end{array}$ & $29035.31 \pm 223.10$ & $28918.91 \pm 243.44$ & $29085.20 \pm 223.10$ & $28885.65 \pm 223.11$ & $29131.76 \pm 228.98$ & $29008.71 \pm 245.77$ & $29035.31 \pm 223.10$ & $28859.03 \pm 259.83$ \\
\hline $\mathbf{P}$ & 0.78 & 0.36 & 0.47 & 0.38 & 0.57 & 0.89 & 0.26 & 0.70 \\
\hline $\begin{array}{l}\text { Female School } \\
\text { students } 1^{\text {st }}\end{array}$ & $29253.58 \pm 288.37$ & $29135.10 \pm 345.64$ & $29309.70 \pm 122.21$ & $29216.16 \pm 133.17$ & $28941.78 \pm 319.37$ & $28810.82 \pm 360.74$ & $28692.33 \pm 177.76$ & $28586.32 \pm 172.82$ \\
\hline $\begin{array}{l}\text { Female } \\
\text { School } \\
\text { students 2 }^{\text {nd }} \\
\end{array}$ & $29253.58 \pm 288.38$ & $29135.09 \pm 345.64$ & $29309.70 \pm 122.18$ & $29216.16 \pm 133.16$ & $28941.76 \pm 319.38$ & $28810.81 \pm 360.72$ & $28692.32 \pm 177.78$ & $28586.31 \pm 172.80$ \\
\hline $\mathbf{v}$ & 0.91 & 0.75 & 0.65 & 0.45 & 0.50 & 0.58 & 0.76 & 0.85 \\
\hline $\begin{array}{l}\text { Male School } \\
\text { students } 1^{\text {st }}\end{array}$ & $29149.35 \pm 250.63$ & $29013.94 \pm 286.38$ & $29348.90 \pm 134.22$ & $29234.87 \pm 107.77$ & $29242.00 \pm 281.37$ & $29177.86 \pm 294.34$ & $28835.76 \pm 107.77$ & $28735.98 \pm 107.77$ \\
\hline $\begin{array}{l}\text { Male School } \\
\text { students2 }^{\text {nd }}\end{array}$ & $29149.34 \pm 250.65$ & $29013.93 \pm 286.39$ & $29348.90 \pm 134.21$ & $29234.87 \pm 107.76$ & $29242.00 \pm 281.37$ & $29177.86 \pm 294.32$ & $28835.76 \pm 107.75$ & $28735.99 \pm 107.77$ \\
\hline $\mathbf{P}$ & 0.67 & 0.25 & 0.26 & 0.38 & 0.56 & 0.69 & 0.57 & 0.50 \\
\hline $\begin{array}{l}\text { Swimming } \\
\text { Female }^{\text {st }}\end{array}$ & $29441.08 \pm 233.90$ & $29534.21 \pm 223.11$ & $29344.63 \pm 209.30$ & $29437.75 \pm 228.98$ & $28839.09 \pm 284.39$ & $29088.53 \pm 320.81$ & $28882.32 \pm 285.64$ & $29028.66 \pm 278.32$ \\
\hline
\end{tabular}




\begin{tabular}{|c|c|c|c|c|c|c|c|c|}
\hline Group Mean $\pm S D$ & $\begin{array}{c}\text { Right Foot } \\
\text { planterflexion }\end{array}$ & $\begin{array}{c}\text { Left Foot } \\
\text { planterflexion }\end{array}$ & $\begin{array}{c}\text { Right Foot } \\
\text { dorsiflexion }\end{array}$ & $\begin{array}{c}\text { Left Foot } \\
\text { dorsiflexion }\end{array}$ & $\begin{array}{c}\text { Right Foot } \\
\text { Eversion }\end{array}$ & $\begin{array}{l}\text { Left Foot } \\
\text { Eversion } \\
\end{array}$ & $\begin{array}{l}\text { Right Foot } \\
\text { Inversion }\end{array}$ & $\begin{array}{l}\text { Left Foot } \\
\text { Inversion }\end{array}$ \\
\hline $\begin{array}{l}\text { Swimming } \\
\text { Female2 }^{\text {nd }}\end{array}$ & $29441.07 \pm 233.89$ & $29534.20 \pm 223.10$ & $29344.63 \pm 209.30$ & $29437.75 \pm 228.98$ & $28839.09 \pm 284.40$ & $29088.53 \pm 320.81$ & $28882.32 \pm 285.64$ & $29028.66 \pm 278.32$ \\
\hline $\mathbf{P}$ & 0.91 & 0.75 & 0.65 & 0.45 & 0.50 & 0.58 & 0.76 & 0.85 \\
\hline $\begin{array}{l}\text { Swimming } \\
\text { Male } 1^{\text {st }}\end{array}$ & $29434.43 \pm 223.11$ & $29517.58 \pm 257.63$ & $29347.95 \pm 206.10$ & $29480.99 \pm 223.48$ & $29311.37 \pm 201.21$ & $29427.78 \pm 233.90$ & $28879.00 \pm 242.85$ & $29135.09 \pm 223.11$ \\
\hline $\begin{array}{l}\text { Swimming } \\
\text { Male 2 }\end{array}$ & $29434.43 \pm 223.11$ & $29517.57 \pm 257.61$ & $29347.95 \pm 206.10$ & $29480.99 \pm 223.48$ & $29311.36 \pm 201.21$ & $29427.77 \pm 233.90$ & $28878.99 \pm 242.86$ & $29135.09 \pm 223.10$ \\
\hline $\mathbf{P}$ & 0.62 & 0.72 & 0.46 & 0.33 & 0.53 & 0.21 & 0.50 & 0.69 \\
\hline $\begin{array}{l}\text { Female } \\
\text { fencing } 1^{\text {st }}\end{array}$ & $29534.21 \pm 223.11$ & $29434.43 \pm 223.11$ & $29384.54 \pm 223.11$ & $29284.76 \pm 223.11$ & $29377.89 \pm 233.90$ & $29281.44 \pm 268.97$ & $29085.21 \pm 223.11$ & $28935.54 \pm 223.11$ \\
\hline $\begin{array}{l}\text { Female } \\
\text { fencing2 }\end{array}$ & $29534.20 \pm 223.10$ & $29434.43 \pm 223.11$ & $29384.54 \pm 223.12$ & $29284.75 \pm 223.11$ & $29377.89 \pm 233.91$ & $29281.43 \pm 268.98$ & $29085.20 \pm 223.10$ & $28935.54 \pm 223.12$ \\
\hline $\mathbf{P}$ & 0.40 & 0.83 & 0.33 & 0.59 & 0.52 & 0.42 & 0.68 & 0.60 \\
\hline $\begin{array}{l}\text { Male fencing } \\
1^{\text {st }}\end{array}$ & $29534.21 \pm 223.11$ & $29391.19 \pm 216.53$ & $29328.00 \pm 233.90$ & $29234.87 \pm 223.11$ & $29361.26 \pm 201.21$ & $29231.55 \pm 228.98$ & $29301.39 \pm 203.67$ & $29115.14 \pm 244.99$ \\
\hline $\begin{array}{l}\text { Male fencing } \\
2^{\text {nd }}\end{array}$ & $29534.20 \pm 223.10$ & $29391.19 \pm 216.54$ & $29327.99 \pm 233.91$ & $29234.86 \pm 223.12$ & $29361.25 \pm 201.21$ & $29231.54 \pm 228.98$ & $29301.39 \pm 203.67$ & $29115.13 \pm 244.98$ \\
\hline $\mathbf{P}$ & 0.44 & 0.62 & 0.29 & 0.88 & 0.25 & 0.51 & 0.87 & 0.88 \\
\hline $\begin{array}{l}\text { Female } \\
\text { Athletics } 1^{\text {st }} \\
\end{array}$ & $29514.25 \pm 244.99$ & $29411.15 \pm 256.38$ & $29484.32 \pm 223.11$ & $29384.54 \pm 223.11$ & $29284.76 \pm 223.11$ & $29188.31 \pm 228.98$ & $29371.24 \pm 244.02$ & $29228.22 \pm 233.90$ \\
\hline $\begin{array}{l}\text { Female } \\
\text { Athletics 2 }^{\text {nd }}\end{array}$ & $29514.25 \pm 244.98$ & $29411.14 \pm 256.39$ & $29484.31 \pm 223.10$ & $29384.54 \pm 223.12$ & $29284.75 \pm 223.11$ & $29188.30 \pm 228.98$ & $29371.23 \pm 244.03$ & $29228.21 \pm 233.90$ \\
\hline $\mathbf{P}$ & 0.69 & 0.63 & 0.47 & 0.22 & 0.94 & 0.84 & 0.45 & 0.65 \\
\hline $\begin{array}{l}\text { Male Athletics } \\
1^{\text {st }}\end{array}$ & $29524.23 \pm 224.06$ & $29434.43 \pm 223.11$ & $29484.32 \pm 223.11$ & $29364.58 \pm 251.43$ & $29484.32 \pm 223.11$ & $29334.65 \pm 223.11$ & $29377.89 \pm 233.90$ & $29268.13 \pm 247.78$ \\
\hline $\begin{array}{l}\text { Male Athletics } \\
2^{\text {nd }}\end{array}$ & $29524.22 \pm 224.06$ & $29434.43 \pm 223.11$ & $29484.31 \pm 223.10$ & $29364.58 \pm 251.43$ & $29484.31 \pm 223.10$ & $29334.65 \pm 223.11$ & $29377.89 \pm 233.91$ & $29268.11 \pm 247.78$ \\
\hline $\mathbf{P}$ & 0.40 & 0.83 & 0.33 & 0.59 & 0.52 & 0.42 & 0.68 & 0.60 \\
\hline 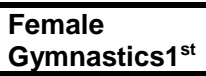 & $28436.65 \pm 223.11$ & $28579.66 \pm 233.90$ & $28735.98 \pm 223.11$ & $28885.65 \pm 223.11$ & $28333.55 \pm 217.84$ & $28473.23 \pm 255.41$ & $28393.41 \pm 224.59$ & $28523.12 \pm 233.59$ \\
\hline $\begin{array}{l}\text { Female } \\
\text { Gymnastics2 }^{n}\end{array}$ & $28446.63 \pm 240.15$ & $28586.32 \pm 244.40$ & $28735.98 \pm 223.10$ & $28888.97 \pm 228.99$ & $28340.20 \pm 228.98$ & $28479.89 \pm 266.58$ & $28400.06 \pm 238.11$ & $28529.78 \pm 247.19$ \\
\hline $\mathbf{P}$ & 0.74 & 0.53 & 0.23 & 0.46 & 0.52 & 0.32 & 0.66 & 0.59 \\
\hline 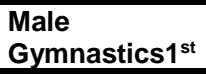 & $29165.03 \pm 160.89$ & $29258.15 \pm 201.21$ & $29058.60 \pm 201.21$ & $29184.98 \pm 270.64$ & $29035.32 \pm 223.11$ & $29118.46 \pm 243.43$ & $28835.76 \pm 223.11$ & $28948.84 \pm 238.12$ \\
\hline $\begin{array}{l}\text { Male } \\
\text { Gymnastics2 }^{n} \\
\text { d }\end{array}$ & $29145.78 \pm 147.98$ & $29238.43 \pm 193.19$ & $29053.13 \pm 216.67$ & $29177.86 \pm 286.16$ & $29046.01 \pm 243.76$ & $29131.53 \pm 262.13$ & $28835.76 \pm 242.84$ & $28942.66 \pm 253.52$ \\
\hline $\mathbf{P}$ & 0.25 & 0.47 & 0.53 & 0.38 & 0.86 & 0.54 & 0.38 & 0.38 \\
\hline $\begin{array}{l}\text { Female } \\
\text { Taekwondo1 }^{\text {st }}\end{array}$ & $29309.70 \pm 122.21$ & $29178.75 \pm 151.88$ & $29658.93 \pm 122.20$ & $29559.15 \pm 122.20$ & $28891.89 \pm 171.66$ & $28785.87 \pm 184.75$ & $29004.14 \pm 133.17$ & $28910.60 \pm 122.21$ \\
\hline $\begin{array}{l}\text { Female } \\
\text { Taekwondo2 }^{\text {nd }}\end{array}$ & $\begin{array}{c}25667.81 \pm 10371.8 \\
4 \\
\end{array}$ & $\begin{array}{c}25555.56 \pm 10326.8 \\
3 \\
\end{array}$ & $\begin{array}{c}25973.39 \pm 10495.3 \\
1 \\
\end{array}$ & $\begin{array}{c}25886.09 \pm 10460.0 \\
3 \\
\end{array}$ & $\begin{array}{c}25243.76 \pm 10200.7 \\
8 \\
\end{array}$ & $\begin{array}{c}25156.45 \pm 10165.9 \\
2 \\
\end{array}$ & $\begin{array}{c}25405.89 \pm 10266.0 \\
1 \\
\end{array}$ & $\begin{array}{c}25318.59 \pm 10230.7 \\
4 \\
\end{array}$ \\
\hline
\end{tabular}




\begin{tabular}{l|c|c|c|c|c|c|c|c}
\hline Group Mean \pm SD & $\begin{array}{c}\text { Right Foot } \\
\text { planterflexion }\end{array}$ & $\begin{array}{c}\text { Left Foot } \\
\text { planterflexion }\end{array}$ & $\begin{array}{c}\text { Right Foot } \\
\text { dorsiflexion }\end{array}$ & $\begin{array}{c}\text { Left Foot } \\
\text { dorsiflexion }\end{array}$ & $\begin{array}{c}\text { Right Foot } \\
\text { Eversion }\end{array}$ & $\begin{array}{c}\text { Left Foot } \\
\text { Eversion }\end{array}$ & $\begin{array}{c}\text { Right Foot } \\
\text { Inversion }\end{array}$ & $\begin{array}{c}\text { Left Foot } \\
\text { Inversion }\end{array}$ \\
\hline $\mathbf{P}$ & 0.55 & 0.79 & 0.30 & 0.37 & 0.63 & 0.39 & 0.39 \\
\hline $\begin{array}{l}\text { Male } \\
\text { Taekwondo1 }\end{array}$ & $29441.56 \pm 120.25$ & $29306.14 \pm 149.27$ & $29726.63 \pm 120.25$ & $29633.98 \pm 107.77$ & $29099.46 \pm 134.22$ & $29006.81 \pm 149.27$ & $29234.87 \pm 107.77$ & $29135.09 \pm 107.77$ \\
\hline $\begin{array}{l}\text { Male } \\
\text { Taekwondo2 }\end{array}$ & $29441.54 \pm 120.25$ & $29306.14 \pm 149.26$ & $29726.63 \pm 120.23$ & $29633.99 \pm 107.77$ & $29099.44 \pm 134.22$ & $29006.80 \pm 149.28$ & $29234.87 \pm 107.76$ & $29135.10 \pm 107.80$ \\
\hline$P$ & 0.74 & 0.53 & 0.23 & 0.46 & & 0.52 & & 0.32 \\
\hline
\end{tabular}

Table (5): Correlations between $1^{\text {st }}$ and $2^{\text {nd }}$ measurements

\begin{tabular}{|c|c|c|c|}
\hline Upper limb & Pearson Correlation & $P$ value & k \\
\hline Pronation Right forearm & $0.358^{\star \star}$ & 0.0001 & 0.95 \\
\hline Pronation Left forearm & $0.455^{\star \star}$ & 0.0001 & 0.90 \\
\hline Supination Right forearm & $-0.274^{\star \star}$ & 0.0001 & 0.94 \\
\hline Supination left forearm & $-0.255^{\star \star}$ & 0.0001 & 0.92 \\
\hline Flexion Right Wrist & $1.000^{\star *}$ & 0.0001 & 0.91 \\
\hline Flexion Left Wrist & $1.000^{* *}$ & 0.0001 & 0.96 \\
\hline Extension Right Wrist & $1.000^{\star \star}$ & 0.0001 & 0.95 \\
\hline Extension Left Wrist & $1.000^{\star \star}$ & 0.0001 & 0.91 \\
\hline Flexion Right Elbow & $1.000^{* *}$ & 0.0001 & 0.89 \\
\hline Flexion Left Elbow & $1.000^{\star \star}$ & 0.0001 & 0.92 \\
\hline Right Shoulder & $1.000^{\star \star}$ & 0.0001 & 0.95 \\
\hline Left Shoulder & $1.000^{* *}$ & 0.0001 & 0.96 \\
\hline \multicolumn{4}{|l|}{ Lower limb } \\
\hline Right Foot planterflexion & $0.364^{\star \star}$ & 0.0001 & 0.91 \\
\hline Left Foot planterflexion & $0.399^{\star \star}$ & 0.0001 & 0.91 \\
\hline Right Foot dorsiflexion & $0.343^{* \star}$ & 0.0001 & 0.92 \\
\hline Left Foot dorsiflexion & $0.370^{\star \star}$ & 0.0001 & 0.96 \\
\hline Right Foot eversion & $0.422^{\star \star}$ & 0.0001 & 0.92 \\
\hline Left Foot eversion & $0.438^{\star \star}$ & 0.0001 & 0.96 \\
\hline Right Foot inversion & $0.395^{\star \star}$ & 0.0001 & 0.90 \\
\hline Left Foot inversion & $0.407^{\text {** }}$ & 0.0001 & 0.91 \\
\hline
\end{tabular}




\section{Discussion}

Hand-held dynamometry (HHD) has never previously been used to examine isometric muscle power. Rate of force development (RFD) is often used for muscle power assessment [13]. The aim of this study was to examine of a new device for assessment of the force of different groups of muscles of the upper limb. Up to our current knowledge, there is no device that can measure the contractile force of different muscles in a limb. This was the reason for inventing this device and therefore we decided to assess of this device on different groups of muscles of upper limb.

To achieve this purpose, muscles of the upper and lower limb of 270 subjects have been examined; 135 athletic and 135 non-athletic (males and females at different age groups and different BMI). The force muscles in each subject were assessed on two sessions 2 weeks apart.

This device has low-cost, portable, and easy-to-use as a clinically-feasible alternative to laboratory-based dynamometry for the assessment of isometric muscle strength.

Previous literature has focused primarily on the assessment and treatment of muscle strength in various clinical populations; however, muscle power is another important consideration. Evidence indicates that in an elderly population, measures of muscle power are more strongly associated with self-reported function and incidence of falls than muscle strength $[4,13]$. Handgrip strength appears to be the most widely used method for the measurement of muscle strength, it is widely used because it can be easily measured in the clinical setting [7].

Clinicians, both from the fields of geriatric medicine and rheumatology, prefer the use of grip strength over chest press and lower limb isokinetic dynamometry as a measure of overall muscle strength[2]. Isometric handgrip strength shows a good correlation with leg strength and also with lower extremity power and calf cross-sectional muscle area [12]. A potential limiting factor for HHD is the lack of widely available software to extract the RFD data [13]. For this reason we have created freely available software program which allows the user to perform load-displacement curve by applying known weights of $1-25 \mathrm{~kg}$ in step of $1 \mathrm{~kg}$. The readings displayed on the LCD screen were monitored and graphed versus the loading values and found to be linearly proportional at the range up to $17 \mathrm{~kg}$.

In the present work, 3 professional assessors shared in the assessment of each group in 2 sessions 2 weeks apart which make the results more accurate and reliable. The assessors were randomly distributed to the different study places. In our experience, sufficient strength levels are required to control the movement of the patient, after which the technique of the assessor is likely to be just as important for obtaining valid results. Although the new device can be applied to any part of the body, yet we applied it to the muscles of the upper limb. This made the study applicable on children and elderly also to prove that there is device that can be applied for the upper limb, we used only pronators, supinators, hand flexors and extensors, elbow extensors and shoulder adductors.

For the treatment of sarcopenia, muscle mass is used. Nevertheless, these methods require massive, costly and non-portable equipment, thereby restricting their use in routine clinical practice. Computer tomography (CT) scanning and magnetic resonance imaging (MRI) allow for precise bone, fat and lean body tissue differentiation and are gold standards for muscle mass evaluation [5].

It is stated that the accuracy of muscle strength values measured using isokinetic dynamometers is high [7], but this instrument is large and expensive; its wider use in clinical practice is therefore limited.

In one study by Marmon [11], the participants were advised to maintain an upright position in an end-sitting position with the hip and knee joints bent at $90^{\circ}$. This test is difficult to be performed by old persons.

It was found that there was a highly significant positive correlation between the 1st and 2 nd measurements, the kappa in all variables was more than 0.70 , it indicates a high association and agreement of the two measurements; the device for the use in different groups of muscles of the upper limb. 
Mentiplay et al. [13] reported that the hip musculature showed the strongest for measures of peak force and RFD. Previous research examining peak force has also found similar results for the assessment of hip strength using HHD in a range of populations [2,5]. HHD in a healthy population has shown poor validity measures of plantarflexor strength in comparison with the electromechanical dynamometer as the gold standard [11]. The group of muscles we have chosen are the muscles that perform the main functions of the upper limb; the prehensive power of the hand and the screwing movement of the forearm.

Starosta et al. [17] in their study on upper limb impairment in the early phase of brain stroke is one of the key problems in rehabilitation. They concluded that neither sex nor the side of the ischemic brain injury influence the muscle force, whereas age determines both muscle force and muscle force deficits. Older post-stroke patients demonstrate fewer deficits in muscle strength than younger ones. In comparison with our device it can assess muscle force in all groups, in all ages and in both sexes.

\section{Conclusions:}

This device for the assessment of muscle force of muscles in the upper limb in both sexes and in different age group. It is recommended to extend the study to include the muscles of other parts of the body not for measurements only but also for training.

Conflicts of Interests

The authors have declared that no competing interests exist.

\section{References:}

1. Bjerk, M., Therese Brovold, Dawn A. Skelton, and Astrid Bergland, (2017) A falls prevention programme to improve quality of life, physical function and falls efficacy in older people receiving home help services: study protocol for a randomised controlled trial. BMC health services research,. Vol 17(1): p. 559.

2. Bruyère, O., C.Beaudart, J.-Y.Reginster, F.Buckinx, D.Schoene, V.Hirani, C.Cooper, J.A.Kanis, R.Rizzoli, E.McCloskey, T.Cederholm, A.Cruz-Jentoft, and E.Freiberger, (2016) Assessment of muscle mass, muscle strength and physical performance in clinical practice: An international survey. Vol. 7. Elsevier European Geriatric Medicine,. p. 243-246.

3. Eleanor Main, L.D., Cardiorespiratory Physiotherapy (2016): Adults and Paediatrics E-Book. Vol. 5. Elsevier Health Sciences. p. 640.

4. Haff, G. Gregory, Ruben, Ryan, Lider, Joshua, Twine, Corey, and Cormie, Prue, (2014) A Comparison of Methods for Determining the Rate of Force Development During Isometric Mid-Thigh Clean Pulls. Vol. 29. Journal of strength and conditioning research / National Strength \& Conditioning Association,. p. 386-395.

5. Kawamori, N., SJ Rossi and BD, Justice, (2006) Peak force and rate of force development during isometric and dynamic mid-thigh clean pulls performed at various intensities. ECU Publications, Vol 20(3), p.483 - 491.

6. Kirkpatrick, L.A. and B.C. Feeney, (2013) A simple guide to IBM SPSS statistics for version 20.0., Belmont, Calif.: Wadsworth, Cengage Learning.

7. Kim, C.R., Y.-J. Jeon, and T. Jeong, (2019) Risk factors associated with low handgrip strength in the older Korean population. PLOS ONE, Vol 14(3): p. e0214612.

8. Kotz S, B.N., Read CB, Vidakovic B. , (2006) Encyclopedia of statistical sciences. 2nd ed. Vol. 16.., Hoboken: N.J.: Wiley-Interscience. 9686.

9. Lieber, R.L. and S.R. Ward, (2011) Skeletal muscle design to meet functional demands. Philos Trans R Soc Lond B Biol Sci,. Vol 366(1570): p. 1466-1476.

10. Madhanagopal, J., Singh, O. P., Sornambikai, S., Sathasivam, K. V., Omar, A. H., Fatihhi, S. J., and Kadir, M. R. A, (2017) Enhanced wide range monotonic piezoresistivity, reliability of ketjenblack/deproteinized natural rubber nanocomposite, and its biomedical application. Journal of Applied Polymer Science,. Vol 134(25): p. 44981- 44991. 
11. Marmon, A.R., Federico Pozzi, Ali H. Alnahdi, and Joseph A. Zeni, (2013) The validity of plantarflexor strength measures obtained through hand-held dynamometry measurements of force. International journal of sports physical therapy,. Vol 8(6): p. 820 - 827.

12. Martins, A.C., Juliana Moreira, Catarina Silva, Joana Silva, Cláudia Tonelo, Daniela Baltazar, Clara Rocha, Telmo Pereira, and Inês Sousa (2018) Multifactorial Screening Tool for Determining Fall Risk in Community-Dwelling Adults Aged 50 Years or Over (FallSensing): Protocol for a Prospective Study. JMIR research protocols,. Vol 7(8): p. e10304-e10304.

13. Mentiplay, B.F., Luke G. Perraton, Kelly J. Bower, Brooke Adair, Yong-Hao Pua, Gavin P. Williams, Rebekah McGaw, and Ross A. Clark, (2015) Assessment of Lower Limb Muscle Strength and Power Using Hand-Held and Fixed Dynamometry: A Reliability and Validity Study. PloS one, Vol 10(10): p. e0140822-e0140822.

14. Nyce, D.S. Linear position sensors (2004) : theory and application.; Available from: http://www.123library.org/book details/?id=25810.

15. PETER A. HUIJING, G.C.B., (2002) Myofascial force transmission: muscle relative position and length determine agonist and synergist muscle force. J Appl Physiol,. Vol 94: p. 20.

16. S Mostert , J.K., (2002) Effects of a short-term exercise training program on aerobic fitness, fatigue, health perception and activity level of subjects with multiple sclerosis. Multiple Sclerosis Journal,. Vol 8(2): p. 8.

17. Starosta, M., J. Kostka, and E. Miller, (2018) Force analysis of shoulder joint muscles in the early phase of brain stroke. Acta of Bioengineering and Biomechanics, . 20: p. 107-113.

18. Tuttle, N., and Jacuinde, G., (2011) Design and Construction of a Novel Low-Cost Device to Provide Feedback on Manually Applied Forces. Journal of Orthopaedic \& Sports Physical Therapy, . Vol 41(3): p. $174-179$.

19. Woolcott, O.O. and R.N. Bergman, (2018) Relative fat mass (RFM) as a new estimator of whole-body fat percentage - A cross-sectional study in American adult individuals. Scientific Reports,. Vol 8(1): p. 10980. 